\title{
Inherent Robustness of Minimal Modeling Discrete-Time Adaptive Control to Flight Anomalies
}

\author{
Mario A. Santillo* and Dennis S. Bernstein ${ }^{\dagger}$ \\ Department of Aerospace Engineering, The University of Michigan, Ann Arbor, MI 48109-2140
}

\begin{abstract}
We present a discrete-time adaptive control law that is effective for systems that are unstable, MIMO, and/or nonminimum phase. The adaptive control algorithm provides guidelines concerning the modeling information needed for implementation. This information includes a sufficient number of Markov parameters to capture the sign of the highfrequency gain as well as the nonminimum-phase zeros. No additional information about the poles or the zeros need be known. We present numerical examples to illustrate the robustness of the algorithm under conditions of uncertainty.
\end{abstract}

\section{Introduction}

Unlike robust control, which fixes the control gains based on a prior, fixed level of modeling uncertainty, adaptive control algorithms tune the feedback gains in response to the true plant and exogenous signals, that is, commands and disturbances. Generally speaking, adaptive controllers require less a priori modeling information than robust controllers, and thus can be viewed as highly parameter-robust control laws. The price paid for the ability of adaptive control laws to operate with limited prior modeling information is the complexity of analyzing and quantifying the stability and performance of the closed-loop system, especially in light of the fact that adaptive control laws, even for linear plants, are nonlinear.

Stability and performance analysis of adaptive control laws often entails restrictive assumptions on the dynamics of the plant. For example, a widely invoked assumption in adaptive control is passivity, ${ }^{1}$ which is restrictive and difficult to verify in practice. A related assumption is that the plant is minimum phase, ${ }^{2,3}$ which may entail the same difficulties. Beyond these assumptions, adaptive control laws are known to be sensitive to unmodeled dynamics and sensor noise, ${ }^{4,5}$ which motivates robust adaptive control laws. ${ }^{6}$

In addition to these basic issues, adaptive control laws may entail unacceptable transients during adaptation, which may be exacerbated by actuator limitations. ${ }^{7-9}$ In fact, adaptive control under extremely limited modeling information such as uncertainty in the high-frequency gain ${ }^{10,11}$ may yield a transient response that exceeds the practical limits of the plant. Therefore, the type and quality of the available modeling information as well as the speed of adaptation must be considered in the analysis and implementation of adaptive control laws. These issues are discussed in Anderson. ${ }^{12}$

Adaptive control laws have been developed in both continuous time and discrete time. In the present paper we consider discrete-time adaptive control laws since these control laws can be implemented directly in embedded code without requiring an intermediate discretization step with potential loss of phase margin. Although discrete-time adaptive control laws are less developed than their continuous-time counterparts, the literature is substantial and growing. ${ }^{2,13-17}$

The goal of the present paper is to present a discrete-time adaptive control law that is effective for nonminimum-phase systems. In Goodwin, et al., ${ }^{2}$ a discrete-time adaptive control law with stability guarantees was developed under a minimum-phase assumption. Extensions given in Hoagg, et al. ${ }^{3}$ based on internal model control ${ }^{18}$ and Lyapunov analysis also invoke this assumption. To circumvent the minimumphase assumption, the zero annihilation periodic control law ${ }^{19}$ uses lifting to move all of the plant's zeros to the origin.

\footnotetext{
${ }^{*}$ National Defense Science and Engineering Graduate Fellow, Department of Aerospace Engineering, The University of Michigan, Ann Arbor, MI 48109-2140

${ }^{\dagger}$ Professor, Department of Aerospace Engineering The University of Michigan, Ann Arbor, MI 48109-2140
} 
The present paper is motivated by the adaptive control laws given by Venugopal and Bernstein ${ }^{20}$ and Hoagg, et al. ${ }^{3}$ The former control law ${ }^{20}$ lacks a proof of stability, but is known numerically to be effective on nonminimum-phase plants without recourse to lifting. Accordingly, we present an adaptive control law based on Venugopal and Bernstein ${ }^{20}$ and Hoagg, et al. ${ }^{3}$ for systems that are unstable, MIMO, and/or nonminimum phase. The adaptive control algorithm provides guidelines concerning the modeling information needed for implementation. This information includes a sufficient number of Markov parameters to capture the sign of the high-frequency gain as well as the nonminimum-phase zeros. No additional information about the poles or the zeros need be known.

The novel feature of this adaptive control law is the use of a retrospective correction filter (RCF). The RCF provides an inner loop to the adaptive control law by modifying the sensor measurements based on the difference between the actual past control inputs and the recomputed past control inputs based on the current control law. This technique is inherent in Venugopal and Bernstein ${ }^{20}$ in the use of the estimated performance variable, but is more fully developed in the present algorithm. Details of the RCF algorithm are given in Santillo and Bernstein. ${ }^{21}$

The goal of the present paper is to demonstrate the RCF adaptive control algorithm's effectiveness in handling nonminimum-phase zeros. We thus present several numerical examples to illustrate the response of the algorithm under modeling error in the relative degree and Markov parameters, measurement noise, and actuator and sensor saturations. To this end we systematically consider a sequence of examples of increasing complexity, ranging from SISO, minimum-phase plants to MIMO, nonminimum-phase plants, including stable and unstable cases. We then revisit these plants under off-nominal conditions, that is, with uncertainty in the required plant modeling information. In each case, we illuminate the role of the weighting parameter $\alpha$, which governs the rate of convergence. Our goal is thus to develop rules of thumb for choosing $\alpha$ based on the level of model fidelity.

These numerical studies show that the RCF adaptive control algorithm is effective for handling nonminimumphase zeros under minimal modeling assumptions. These studies also provide guidance into the choice of $\alpha$ for stable response and acceptable transient behavior. This guidance can provide the basis for Lyapunov-based stability and performance analysis in future work.

\section{Problem Formulation}

Consider the MIMO discrete-time system

$$
\begin{aligned}
x(k+1) & =A x(k)+B u(k)+D_{1} w(k), \\
y(k) & =C x(k)+D_{2} w(k), \\
z(k) & =E_{1} x(k)+E_{0} w(k),
\end{aligned}
$$

where $x(k) \in \mathbb{R}^{n}, y(k) \in \mathbb{R}^{l_{y}}, z(k) \in \mathbb{R}^{l_{z}}, u(k) \in \mathbb{R}^{l_{u}}, w(k) \in \mathbb{R}^{l_{w}}$, and $k \geq 0$. Our goal is to develop an adaptive output feedback controller under which the performance variable $z$ is minimized in the presence of the exogenous signal $w$. Note that $w$ can represent either a command signal to be followed, an external disturbance to be rejected, or both. For example, if $D_{1}=0$ and $E_{0} \neq 0$, then the objective is to have the output $E_{1} x$ follow the command signal $-E_{0} w$. On the other hand, if $D_{1} \neq 0$ and $E_{0}=0$, then the objective is to reject the disturbance $w$ from the performance measurement $E_{1} x$. The combined command following and disturbance rejection problem is addressed when $D_{1}$ and $E_{0}$ are block matrices. More precisely, if $D_{1}=\left[\begin{array}{cc}\hat{D}_{1} & 0\end{array}\right], E_{0}=\left[\begin{array}{cc}0 & \hat{E}_{0}\end{array}\right]$, and $w(k)=\left[\begin{array}{c}w_{1}(k) \\ w_{2}(k)\end{array}\right]$, then the objective is to have $E_{1} x$ follow the command $-\hat{E}_{0} w_{2}$ while rejecting the disturbance $w_{1}$. Lastly, if $D_{1}$ and $E_{0}$ are empty matrices, then the objective is output stabilization, that is, convergence of $z$ to zero.

Model reference adaptive control (MRAC) is a special case of (1)-(3) where $z \triangleq y_{1}-y_{\mathrm{m}}$ is the difference between the measured output of the plant $G$ and reference model $G_{\mathrm{m}}$. For MRAC, the exogenous command $w$ is available to the controller as an additional measurement variable $y_{2}$, as shown in Figure 1.

\section{Controller Construction}

In this section we give a brief overview of an adaptive control algorithm for the general control problem represented by (1)-(3). The algorithm is derived from the work of Venugopal and Bernstein ${ }^{20}$ and Hoagg, 


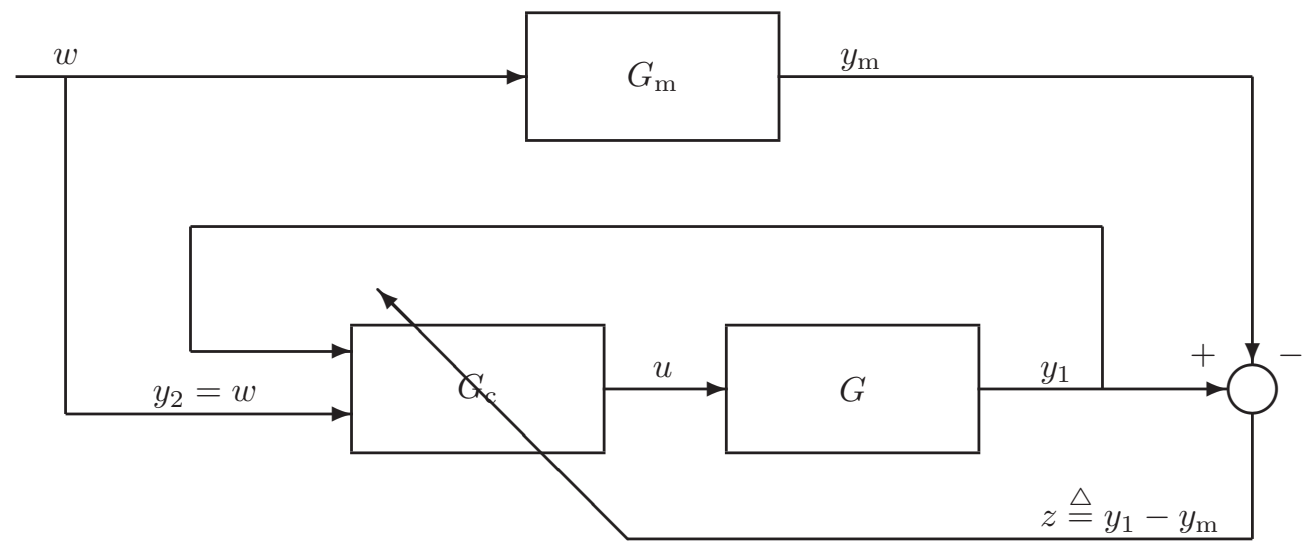

Figure 1. Model reference adaptive control problem.

et al. ${ }^{3}$ The full details of the algorithm are presented in Santillo and Bernstein. ${ }^{21}$

We use a strictly proper time-series controller of order $n_{\mathrm{c}}$, such that the control $u(k)$ is given by

$$
u(k)=\sum_{i=1}^{n_{\mathrm{c}}} M_{i}(k) u(k-i)+\sum_{i=1}^{n_{\mathrm{c}}} N_{i}(k) y(k-i)
$$

where, for all $i=1, \ldots, n_{\mathrm{c}}, M_{i} \in \mathbb{R}^{l_{u} \times l_{u}}$ and $N_{i} \in \mathbb{R}^{l_{u} \times l_{y}}$ are given by an adaptive update law. The control can be expressed as

$$
u(k)=\theta(k) \phi(k),
$$

where

$$
\theta(k) \triangleq\left[\begin{array}{llllll}
N_{1}(k) & \cdots & N_{n_{\mathrm{c}}}(k) & M_{1}(k) & \cdots & M_{n_{\mathrm{c}}}(k)
\end{array}\right] \in \mathbb{R}^{l_{u} \times n_{\mathrm{c}}\left(l_{u}+l_{y}\right)}
$$

is the controller parameter block matrix, and the regressor vector $\phi(k)$ is given by

$$
\phi(k) \triangleq\left[\begin{array}{c}
y(k-1) \\
\vdots \\
y\left(k-n_{\mathrm{c}}\right) \\
u(k-1) \\
\vdots \\
u\left(k-n_{\mathrm{c}}\right)
\end{array}\right] \in \mathbb{R}^{n_{\mathrm{c}}\left(l_{u}+l_{y}\right)} .
$$

For positive integers $p$ and $\mu$, we define the extended performance vector $Z(k)$, and the extended control vector $U(k)$ by

$$
Z(k) \triangleq\left[\begin{array}{c}
z(k) \\
\vdots \\
z(k-p+1)
\end{array}\right], \quad U(k) \triangleq\left[\begin{array}{c}
u(k) \\
\vdots \\
u\left(k-p_{\mathrm{c}}+1\right)
\end{array}\right]
$$

where $p_{\mathrm{c}} \triangleq \mu+p$.

From (5), it follows that the extended control vector $U(k)$ can be written as

$$
U(k) \triangleq \sum_{i=1}^{p_{\mathrm{c}}} L_{i} \theta(k-i+1) \phi(k-i+1)
$$


where

$$
L_{i} \triangleq\left[\begin{array}{c}
0_{(i-1) l_{u} \times l_{u}} \\
I_{l_{u}} \\
0_{\left(p_{\mathrm{c}}-i\right) l_{u} \times l_{u}}
\end{array}\right] \in \mathbb{R}^{p_{c} l_{u} \times l_{u}} .
$$

We define the surrogate performance vector $\hat{Z}(\hat{\theta}(k), k)$ by

$$
\hat{Z}(\hat{\theta}(k), k) \triangleq Z(k)-\bar{B}_{z u}(U(k)-\hat{U}(k)),
$$

where $\hat{U}(k) \triangleq \sum_{i=1}^{p_{c}} L_{i} \hat{\theta}(k) \phi(k-i+1), \hat{\theta}(k) \in \mathbb{R}^{l_{u} \times n_{\mathrm{c}}\left(l_{u}+l_{y}\right)}$ is the surrogate controller parameter block matrix, and the block-Toeplitz surrogate control matrix $\bar{B}_{z u} \in \mathbb{R}^{p l_{z} \times p_{c} l_{u}}$ is given by

$$
\bar{B}_{z u} \triangleq\left[\begin{array}{ccccccccc}
0_{l_{z} \times l_{u}} & \cdots & 0_{l_{z} \times l_{u}} & H_{d} & \cdots & H_{\mu} & 0_{l_{z} \times l_{u}} & \cdots & 0_{l_{z} \times l_{u}} \\
0_{l_{z} \times l_{u}} & \ddots & & \ddots & \ddots & & \ddots & \ddots & \vdots \\
\vdots & \ddots & \ddots & & \ddots & \ddots & & \ddots & 0_{l_{z} \times l_{u}} \\
0_{l_{z} \times l_{u}} & \cdots & 0_{l_{z} \times l_{u}} & 0_{l_{z} \times l_{u}} & \cdots & 0_{l_{z} \times l_{u}} & H_{d} & \cdots & H_{\mu}
\end{array}\right]
$$

where the relative degree $d$ is the smallest positive integer $i$ such that the $i$ th Markov parameter $H_{i} \triangleq$ $E_{1} A^{i-1} B$ is nonzero. The leading zeros in the first row of $\bar{B}_{z u}$ account for the nonzero relative degree $d$. The algorithm places no constraints on either the value of $d$ or the rank of $H_{d}$ or $\bar{B}_{z u}$.

The adaptive update law presented in Santillo and Bernstein ${ }^{21}$ depends on a time-varying weighting parameter $\alpha(k) \in(0, \infty)$, referred to as the learning rate since it affects convergence speed of the adaptive control algorithm. As $\alpha$ is increased, convergence speed is lowered. Likewise, as $\alpha$ is decreased, converge speed is raised. For the nominal examples in the next section, $\alpha$ is a small number. In the off-nominal examples presented later, $\alpha$ is increased to account for the lack of model fidelity.

The novel feature of the adaptive control algorithm (5) is the use of the retrospective correction filter (RCF) (11), as shown in Figure 2 for $p=1$. The RCF provides an inner loop to the adaptive control law by modifying the performance variable $Z(k)$ based on the difference between the actual past control inputs $U(k)$ and the recomputed past control inputs based on the current control law $\hat{U}(k)$.

\section{Numerical Examples - Nominal Case}

We now present numerical examples to illustrate the response of the RCF adaptive control algorithm under nominal conditions. We consider a sequence of examples of increasing complexity, ranging from SISO, minimum-phase plants to MIMO, nonminimum-phase plants, including stable and unstable cases. Each plant can be viewed as a sampled-data discretization of a continuous-time plant sampled at $T_{\mathrm{s}}=0.01 \mathrm{sec}$. All of the following examples assume $z=y$.

Unless otherwise noted, each example is taken to be a disturbance rejection simulation, that is, $E_{0}=0$, with unknown sinusoidal disturbance given by

$$
w(k)=\left[\begin{array}{c}
\sin 2 \pi \nu_{1} k T_{\mathrm{s}} \\
\sin 2 \pi \nu_{2} k T_{\mathrm{s}}
\end{array}\right],
$$

where $\nu_{1}=5 \mathrm{~Hz}$ and $\nu_{2}=13 \mathrm{~Hz}$. The RCF adaptive control algorithm requires no information about $w$. With each plant realized in controllable canonical form, we take $D_{1}=\left[\begin{array}{c}I_{2} \\ 0\end{array}\right]$, and, therefore, the disturbance is not matched.

Example IV.1 (SISO, Minimum Phase, Stable Plant). Consider a plant with poles $\{0.5 \pm 0.5\},-0.5 \pm$ $0.5 \mathrm{\jmath}, \pm 0.9, \pm 0.7 \mathrm{\jmath}\}$ and zeros $\{0.3 \pm 0.7 \mathrm{\jmath},-0.7 \pm 0.3 \mathrm{\jmath}, 0.5\}$. We take $n_{\mathrm{c}}=15, p=1, \mu=3$, and $\alpha=25$. The closed-loop response is shown in Figure 3. The control is turned on at $t=2 \mathrm{sec}$, and the performance variable reduces to zero within $1 \mathrm{sec}$. The control algorithm converges to an internal model controller with high gain at the disturbance frequency, as seen in Figure 4. 


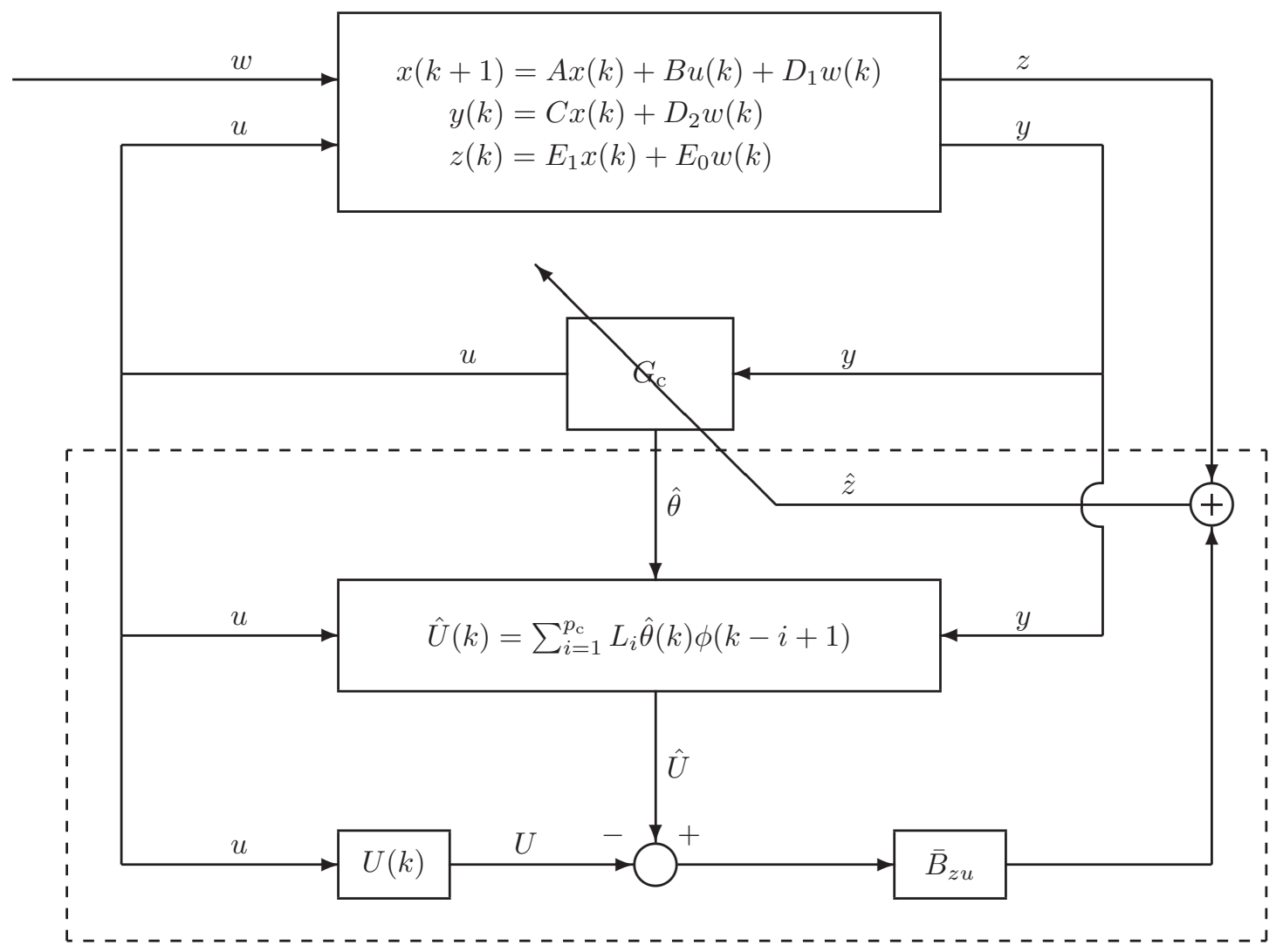

Figure 2. Closed-loop system including adaptive control algorithm with the retrospective correction filter (dash box) for $p=1$.
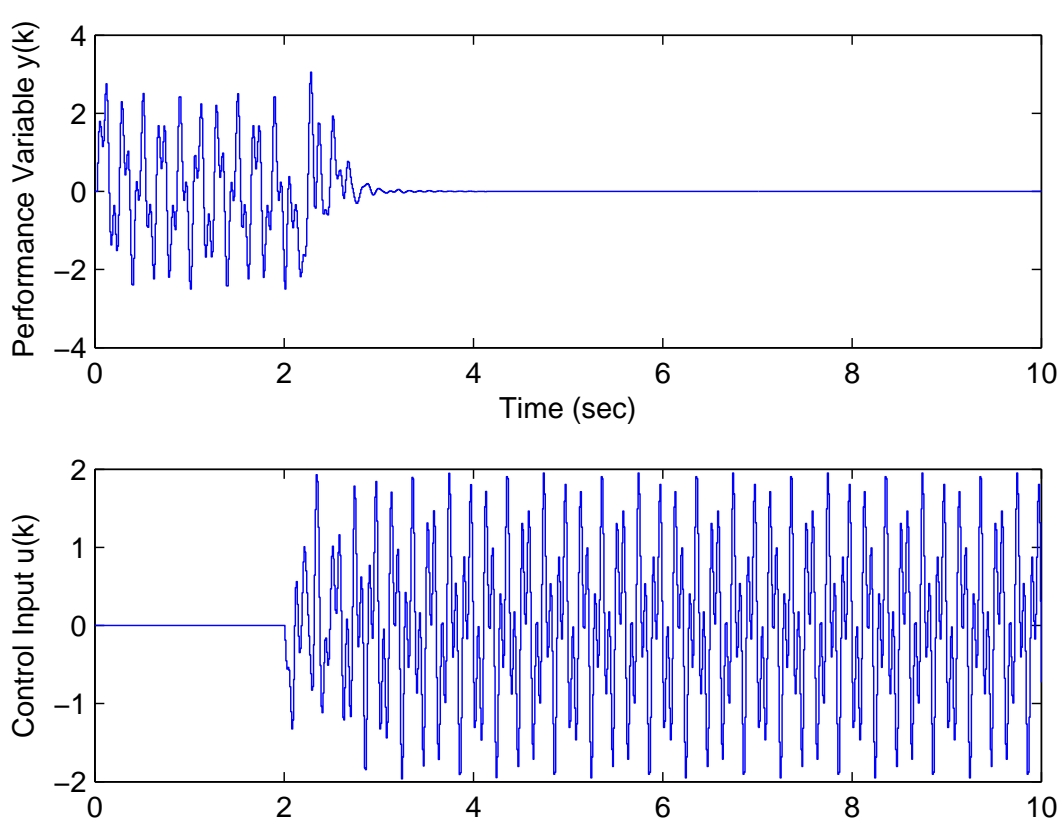

Figure 3. Closed-loop disturbance rejection response for a stable, minimum phase, SISO plant. The control is turned on at $t=2 \mathrm{sec}$. The controller order is $n_{\mathrm{c}}=15$ with parameters $p=1, \mu=3, \alpha=25$. 


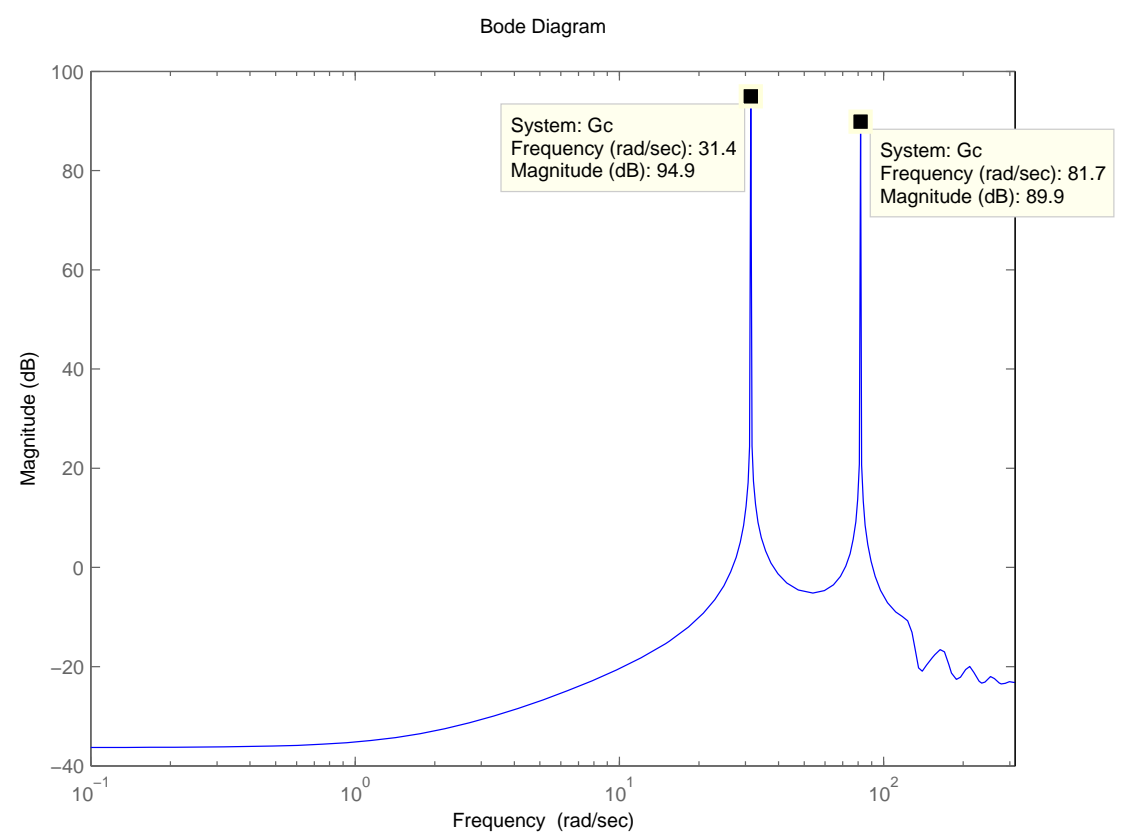

Figure 4. Bode magnitude plot of the adaptive controller at $t=10$ sec. The adaptive controller places poles at the disturbance frequencies $\nu_{1}=5 \mathrm{~Hz}$ and $\nu_{2}=13 \mathrm{~Hz}$. The controller magnitude $\left|G_{\mathrm{c}}\left(e^{\jmath \omega T_{\mathrm{S}}}\right)\right|$ is plotted for $\omega$ up to the Nyquist frequency $\omega_{\mathrm{Nyq}}=\frac{\pi}{T_{\mathrm{s}}}=314 \mathrm{rad} / \mathrm{sec}$.

Example IV.2 (SISO, Nonminimum Phase, Stable Plant). Consider a plant with poles $\{0.5 \pm 0.5 \mathrm{j},-0.5 \pm$ $0.5 \mathrm{~J}, \pm 0.9, \pm 0.7 \mathrm{\jmath}\}$ and zeros $\{0.3 \pm 0.7 \mathrm{\jmath},-0.7 \pm 0.3 \mathrm{~J}, 2\}$. We take $n_{\mathrm{c}}=15, p=1, \mu=7$, and $\alpha=25$. The closed-loop response is shown in Figure 5 . The control is turned on at $t=2 \mathrm{sec}$, and, after a slight transient, the performance variable reduces to zero.
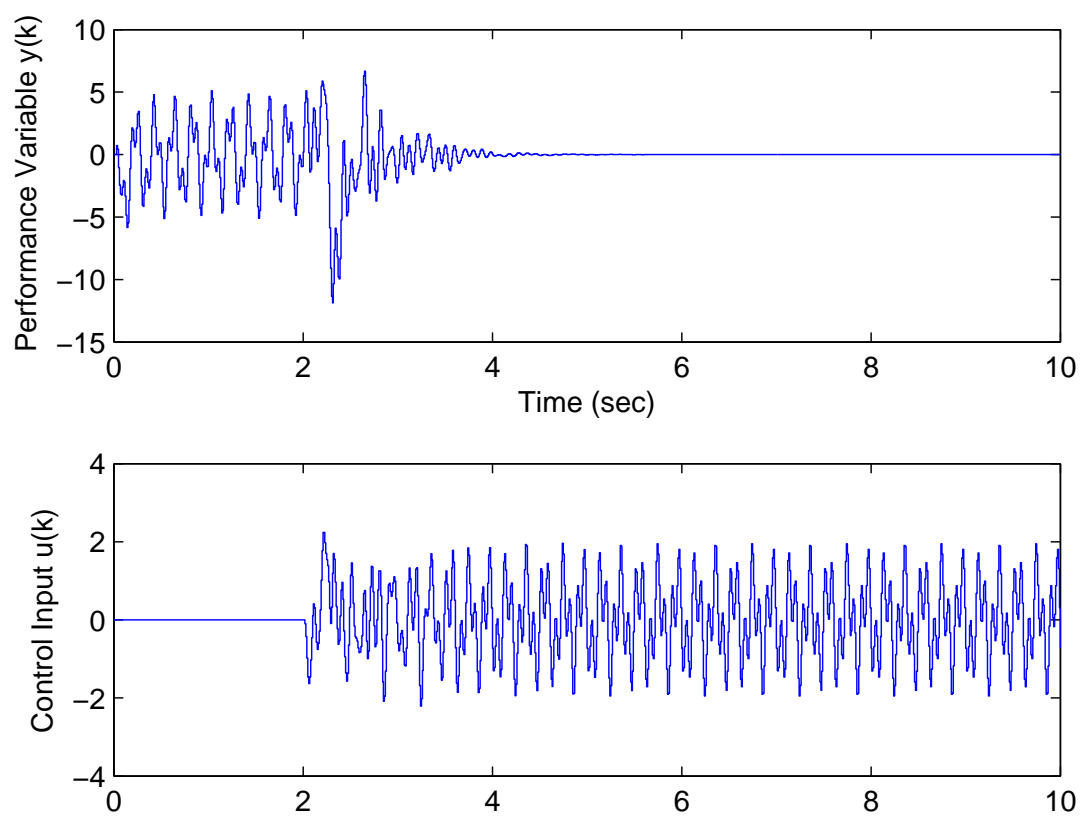

Figure 5. Closed-loop disturbance rejection response for a stable, nonminimum phase, SISO plant. The control is turned on at $t=2 \mathrm{sec}$. The controller order is $n_{\mathrm{c}}=15$ with parameters $p=1, \mu=7, \alpha=25$. 
Example IV.3 (SISO, Minimum Phase, Unstable Plant). Consider a plant with poles $\{0.5 \pm 0.5\},-0.5 \pm$ $0.5 \jmath, \pm 1.04,0.1 \pm 1.025\}\}$ and zeros $\{0.3 \pm 0.7 \jmath,-0.7 \pm 0.3 \jmath, 0.5\}$. We take $n_{\mathrm{c}}=15, p=1, \mu=10$, and $\alpha=25$. The closed-loop response is shown in Figure 6. The control is turned on at $t=2 \mathrm{sec}$, and, after a transient, the performance variable reduces to zero.
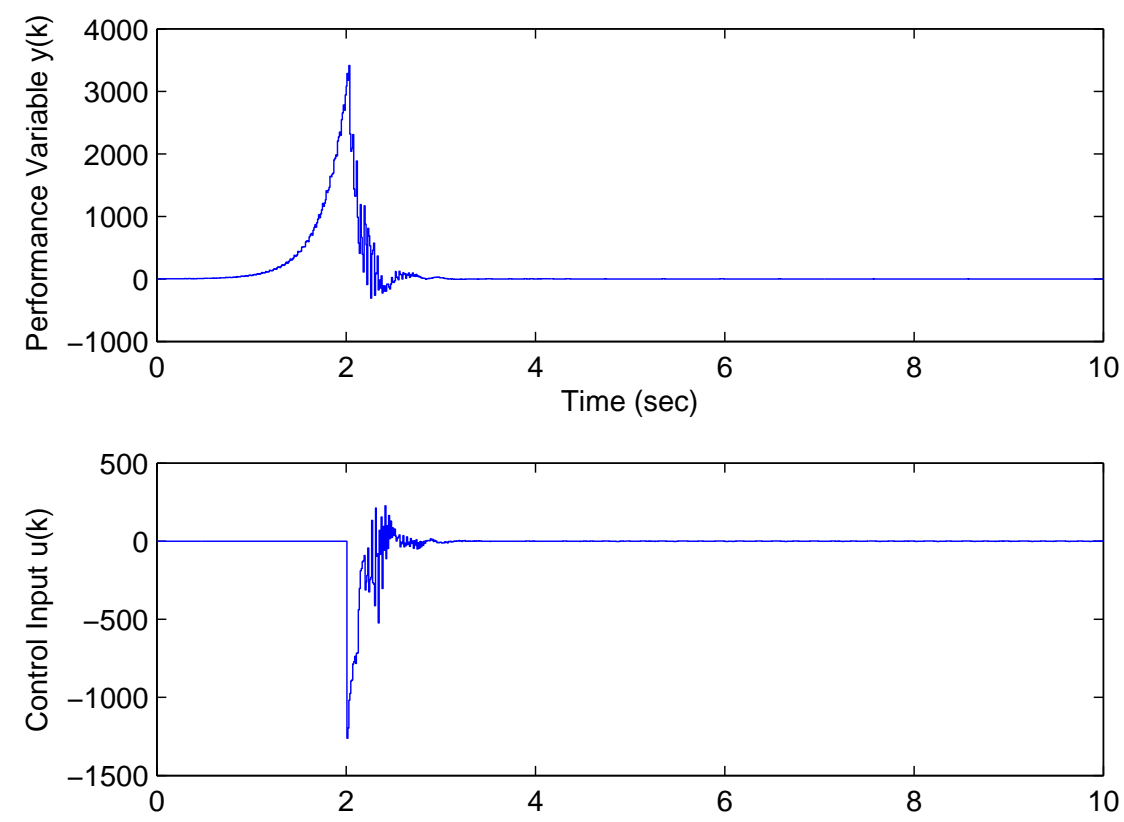

Figure 6. Closed-loop disturbance rejection response for an unstable, minimum phase, SISO plant. The control is turned on at $t=2 \mathrm{sec}$. The controller order is $n_{\mathrm{c}}=15$ with parameters $p=1, \mu=10, \alpha=25$.

Example IV.4 (MIMO, Minimum Phase, Stable Plant). Consider a two-input, two-output plant with poles $\{-0.5 \pm 0.5 \jmath, 0.9, \pm 0.7 \jmath,-0.5 \pm 0.5 \jmath, 0.9, \pm 0.7 \jmath\}$ and transmission zeros $\{0.3 \pm 0.7 \jmath, 0.5,0.5\}$. We take $n_{\mathrm{c}}=15$, $p=1, \mu=10$, and $\alpha=1$. The closed-loop response is shown in Figure 7. The control is turned on at $t=2 \mathrm{sec}$, and the performance variable reduces to zero.

Example IV.5 (MIMO, Nonminimum Phase, Stable Plant). Consider a two-input, two-output plant with poles $\{-0.5 \pm 0.5 \jmath, 0.9,-0.5 \pm 0.5 \jmath, 0.9\}$ and transmission zero $\{2\}$. We take $n_{\mathrm{c}}=20, p=1, \mu=6$, and $\alpha=1$. The closed-loop response is shown in Figure 8. The control is turned on at $t=2 \mathrm{sec}$, and, after a slight transient, the performance variable reduces to zero.

Example IV.6 (Ex. IV.1 with Command Following and Disturbance Rejection). We consider a combined step-command following and disturbance rejection problem with command and disturbance given by

$$
w(k)=\left[\begin{array}{c}
w_{1}(k) \\
w_{2}(k)
\end{array}\right]=\left[\begin{array}{c}
\sin 2 \pi \nu_{1} k T_{\mathrm{s}} \\
5
\end{array}\right]
$$

With the plant realized in controllable canonical form, we take $D_{1}=\left[\begin{array}{ll}1 & 0 \\ 0 & 0\end{array}\right]$ and $E_{0}=\left[\begin{array}{ll}0 & -1\end{array}\right]$. Therefore, $w_{1}$ is the disturbance to be rejected, while $w_{2}$ is the command to be followed.

We take $n_{\mathrm{c}}=20, p=1, \mu=3$, and $\alpha=50$. The closed-loop response is shown in Figure 9 . The control is turned on at $t=2 \mathrm{sec}$, and the performance variable reduces to zero, that is, the disturbance $w_{1}$ is rejected while the command $w_{2}$ is followed.

Example IV.7 (Command Following with Unstable Plant). We consider a double integrator plant with command given by $w(k)=1$. With the plant realized in controllable canonical form, we take $D_{1}=0$ and $E_{0}=-1$. 

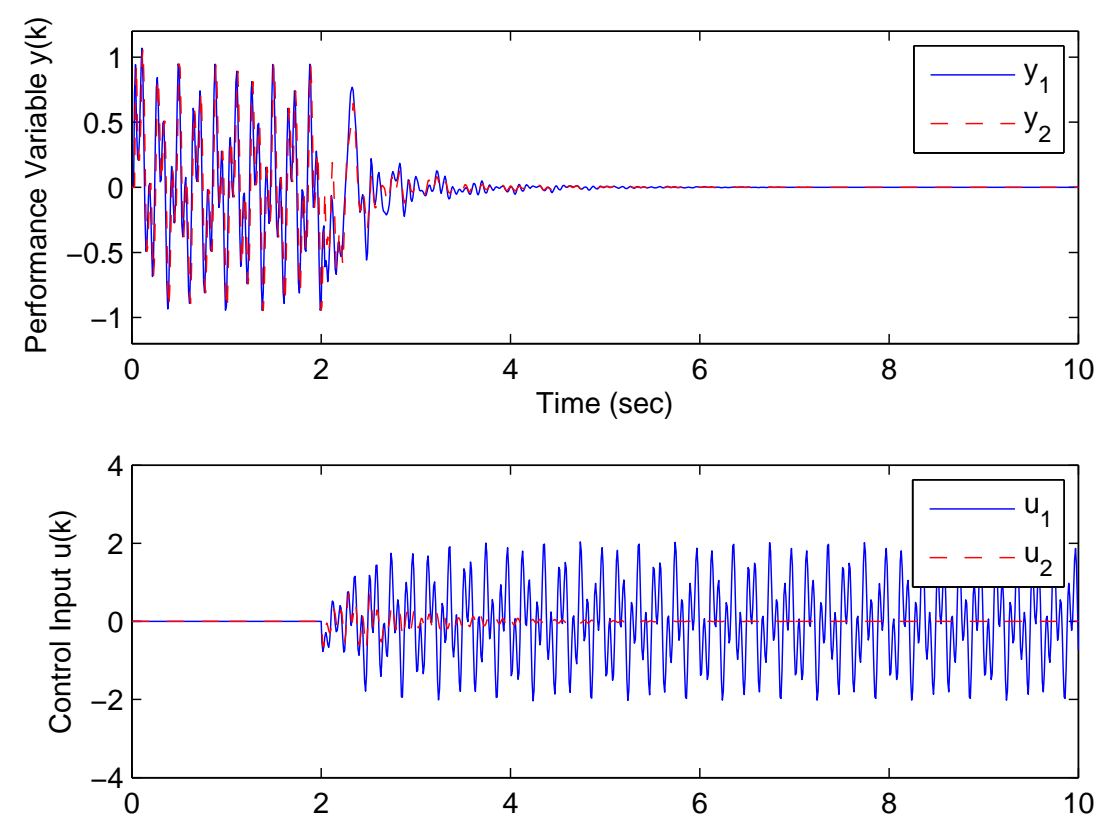

Figure 7. Closed-loop disturbance rejection response for a stable, minimum phase, two-input two-output plant. The control is turned on at $t=2 \mathrm{sec}$. The controller order is $n_{\mathrm{c}}=15$ with parameters $p=1, \mu=10, \alpha=1$.
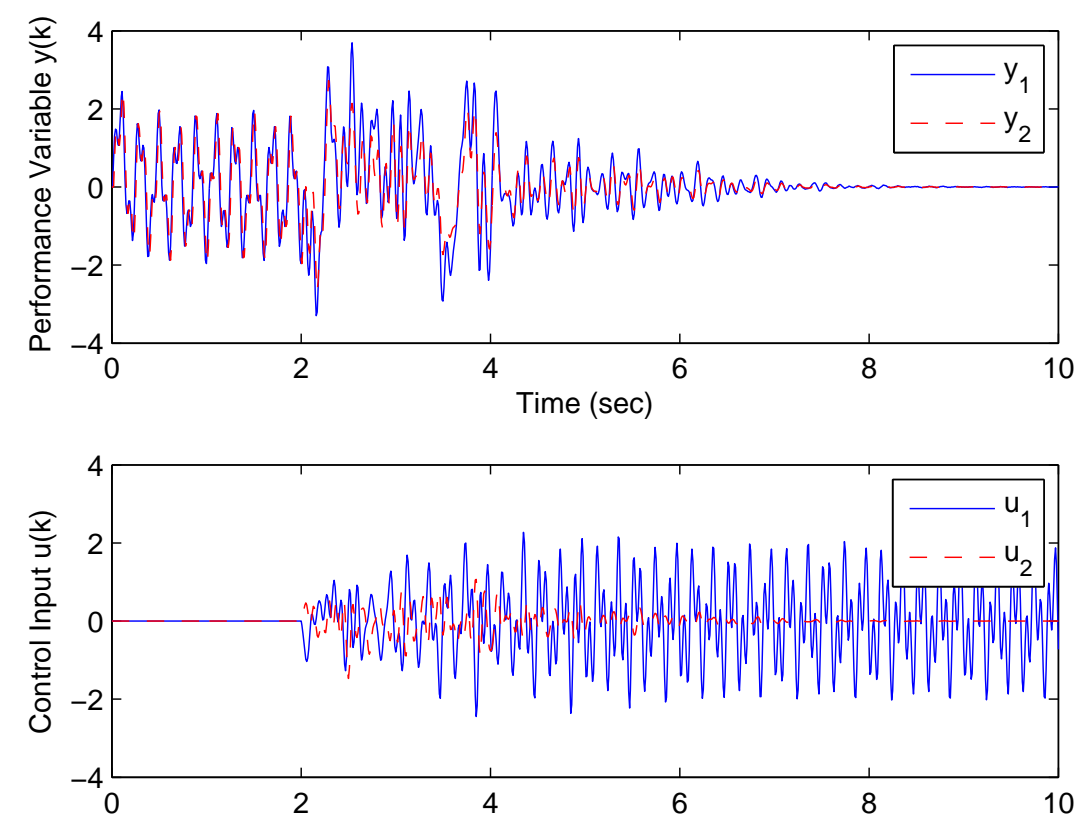

Figure 8. Closed-loop disturbance rejection response for a stable, nonminimum phase, two-input two-output plant. The control is turned on at $t=2 \mathrm{sec}$. The controller order is $n_{\mathrm{c}}=20$ with parameters $p=1, \mu=6, \alpha=1$.

The SISO plant is unstable and minimum phase with poles $\{0.5 \pm 0.5 \jmath,-0.5 \pm 0.5 \jmath, 1,1\}$ and zeros $\{0.3 \pm 0.7 \jmath, 0.5\}$. We take $n_{\mathrm{c}}=10, p=5, \mu=10$, and $\alpha=5$. The closed-loop response is shown in Figure 10. The control is turned on at $t=2 \mathrm{sec}$, and, after a transient, the performance variable reduces to zero, that is, the step-command $w$ is followed. 

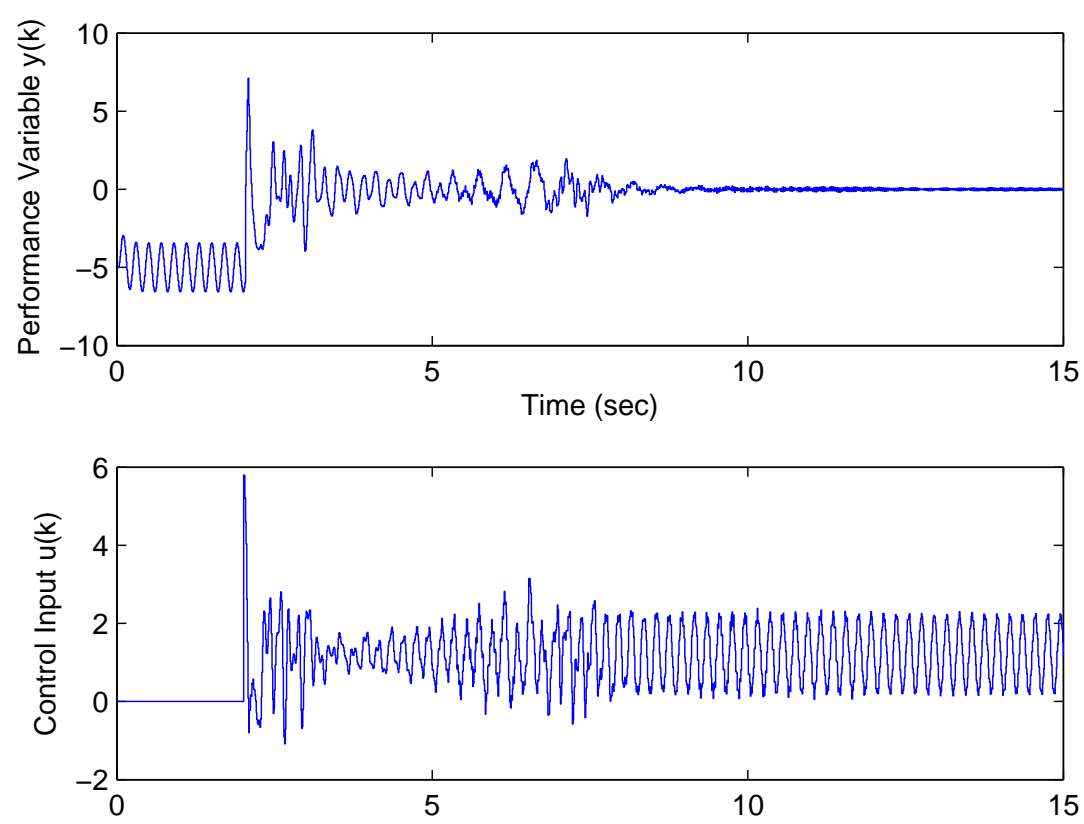

Figure 9. Closed-loop response for a stable, minimum phase, SISO plant with a step command and sinusoidal disturbance. The control is turned on at $t=2 \mathrm{sec}$. The controller order is $n_{\mathrm{c}}=20$ with parameters $p=1, \mu=3, \alpha=50$.
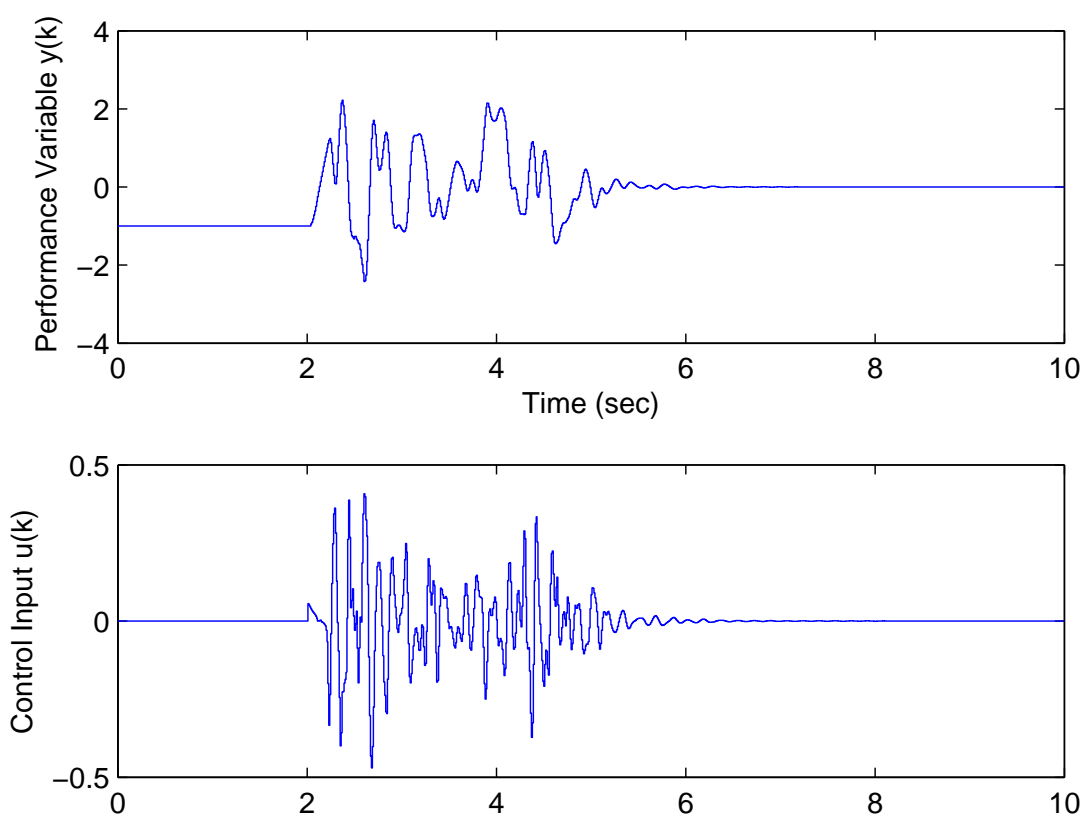

Figure 10. Closed-loop response for an unstable, minimum phase, SISO plant with a step command. The control is turned on at $t=2 \mathrm{sec}$. The controller order is $n_{\mathrm{c}}=10$ with parameters $p=5, \mu=10, \alpha=5$.

\section{Numerical Examples - Off-nominal Cases}

We now present numerical examples to illustrate the response of the RCF adaptive control algorithm under modeling errors in the relative degree and Markov parameters, measurement noise, and actuator and sensor saturations. Therefore, we revisit examples from the previous section under off-nominal conditions, 
that is, with uncertainty in the required plant modeling information. In each case, we illuminate the role of the learning rate $\alpha$, which governs the rate of convergence. Our goal is thus to develop rules of thumb for choosing $\alpha$ based on the level of model fidelity. Each example is taken to be a disturbance rejection simulation with $z=y$, as presented in Section IV.

Example V.1 (Ex. IV.2 with Relative Degree Error and Unknown Latency). Consider model error in the relative degree. The system has relative degree $d=3$.

First, for controller implementation, we use the erroneous $\hat{d}=2$. We take $n_{\mathrm{c}}=15, p=1, \mu=10$, and $\alpha=1000$. The closed-loop response is shown in Figure 11. The control is turned on at $t=2$ sec, and the performance variable reduces to zero.
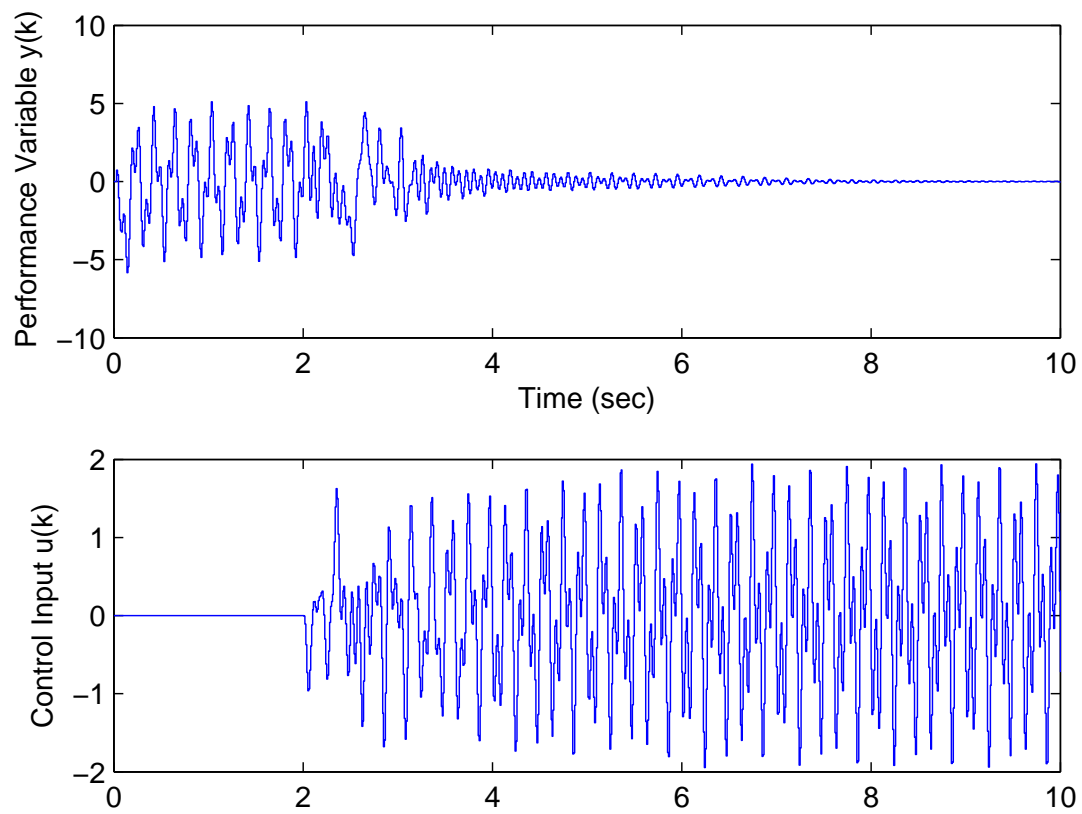

Figure 11. Closed-loop disturbance rejection response for a stable, nonminimum phase, relative degree $d=3$ SISO plant where the controller is created assuming the plant has relative degree $\hat{d}=2$. The control is turned on at $t=2$ sec. The controller order is $n_{c}=15$ with parameters $p=1, \mu=10, \alpha=1000$. To compensate for uncertainty in the relative degree $d$, the tuning parameter $\alpha$ is increased to slow down the adaptation.

Now let $\hat{d}=4$. We take $n_{\mathrm{c}}=15, p=1, \mu=10$, and $\alpha=1000$. The closed-loop response is shown in Figure 12. The control is turned on at $t=2 \mathrm{sec}$, and the performance variable converges to zero.

These simulations show that the adaptive controller is sensitive to errors in relative degree, which is equivalent to an unknown latency, that is, implementation delay. However, the effect of a known latency of $l$ steps can be addressed by simply replacing $d$ by $d+l$ in the construction of $\bar{B}_{z u}$.

Example V.2 (Ex. IV.1 with Uncertain $H_{d}$ ). We now assess the algorithm's robustness to knowledge of the first nonzero Markov parameter $H_{d}$. The first nonzero Markov parameter is $H_{3}=1$.

We first assume that the first nonzero Markov parameter is $\hat{H}_{3}=0.05 H_{3}$. We take $n_{\mathrm{c}}=15, p=1, \mu=3$, and $\alpha=25$. The closed-loop response is shown in Figure 13. The control is turned on at $t=2$ sec, and the performance variable converges within 6 sec.

Now, we assume that the first nonzero Markov parameter is $\hat{H}_{3}=20 H_{3}$. We take $n_{\mathrm{c}}=15, p=1, \mu=3$, and $\alpha=25$. The closed-loop response is shown in Figure 14. The control is turned on at $t=2$ sec, and the performance variable converges to zero.

In the case where the sign of the high-frequency gain is wrong, that is, $\hat{H}_{3}=-H_{3}$, the weighting parameter $\alpha$ must be chosen so large that the adaptation is essentially stopped. As the fidelity of $H_{d}$ decreases, convergence is slowed. From these results it is seen that increasing error in $H_{d}$ is equivalent to increasing $\alpha$, and thus slowing down the convergence.

Example V.3 (Noisy Markov Parameters). We investigate model error in the Markov parameters. 

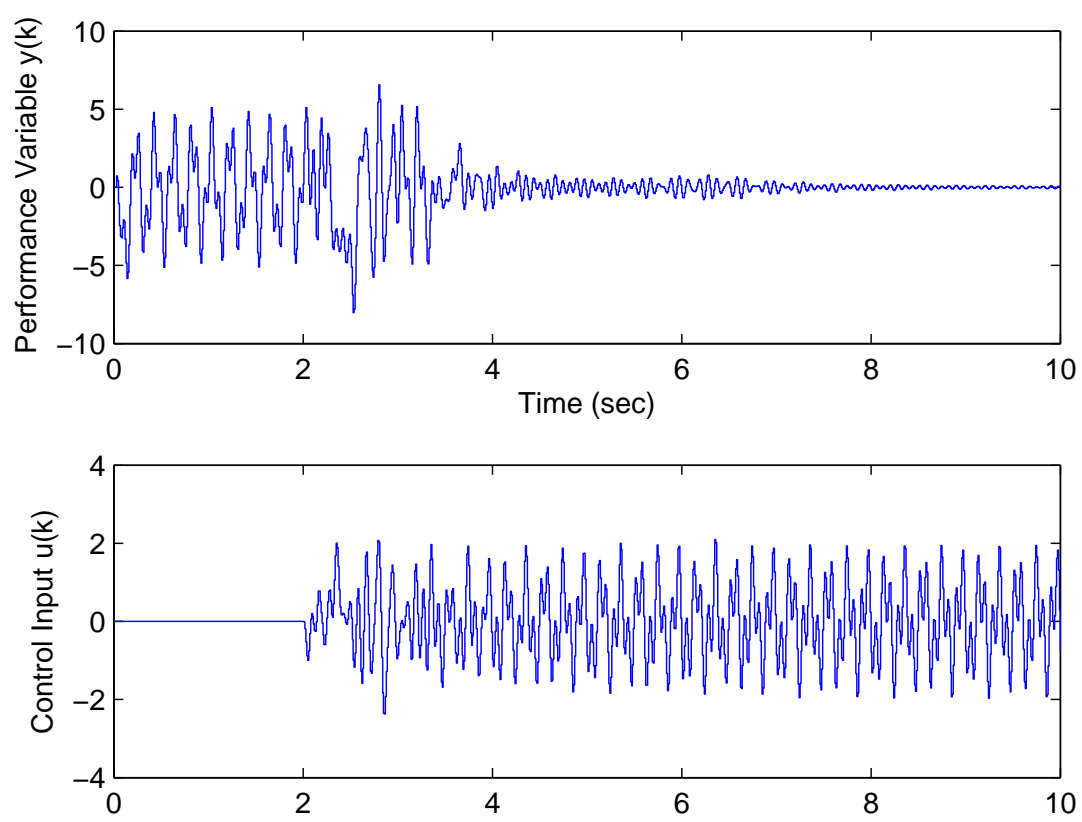

Figure 12. Closed-loop disturbance rejection response for a stable, nonminimum phase, relative degree $d=3$ SISO plant where the controller is created assuming the plant has relative degree $\hat{d}=4$. The control is turned on at $t=2$ sec. The controller order is $n_{\mathrm{c}}=15$ with parameters $p=1, \mu=10, \alpha=1000$.
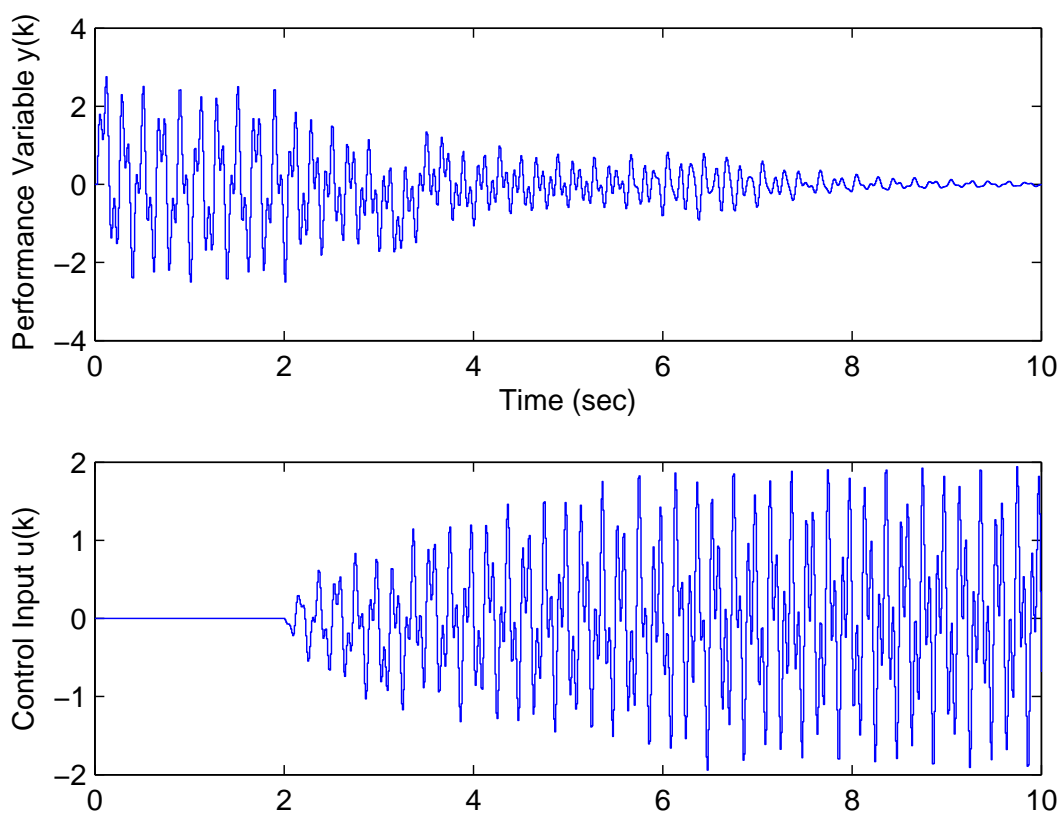

Figure 13. Closed-loop disturbance rejection response for a stable, minimum phase, SISO plant with $H_{d}=1$ where the controller is created with $\hat{H}_{d}=0.05$. The control is turned on at $t=2 \mathrm{sec}$. The controller order is $n_{\mathrm{c}}=15$ with parameters $p=1, \mu=3, \alpha=25$. With $H_{d}$ underestimated, the closed-loop converges more slowly than in the nominal case.

First, consider Example IV.1. The system has relative degree $d=3$ with $H_{3}=1$. For controller implementation, we perturb each Markov parameter $H_{i}, i=1 \ldots \mu$, by adding zero-mean Gaussian white 

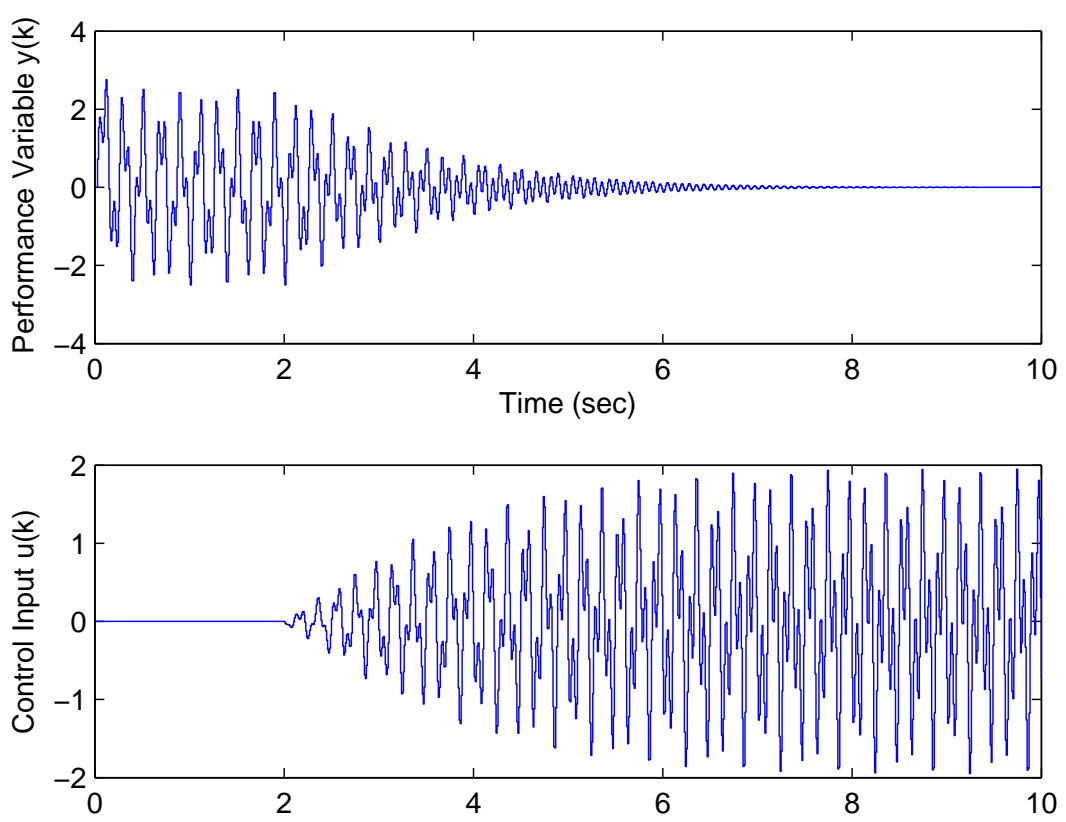

Figure 14. Closed-loop disturbance rejection response for a stable, minimum phase, SISO plant with $H_{d}=1$ where the controller is created with $\hat{H}_{d}=20$. The control is turned on at $t=2 \mathrm{sec}$. The controller order is $n_{\mathrm{c}}=15$ with parameters $p=1, \mu=3, \alpha=25$. With $H_{d}$ overestimated, the closed-loop converges more slowly than in the nominal case.

noise with standard deviation $\sigma=0.25$. We take $n_{\mathrm{c}}=15, p=1, \mu=3$, and $\alpha=25$. The closed-loop response is shown in Figure 15. The control is turned on at $t=2 \mathrm{sec}$, and the performance variable reduces to zero.
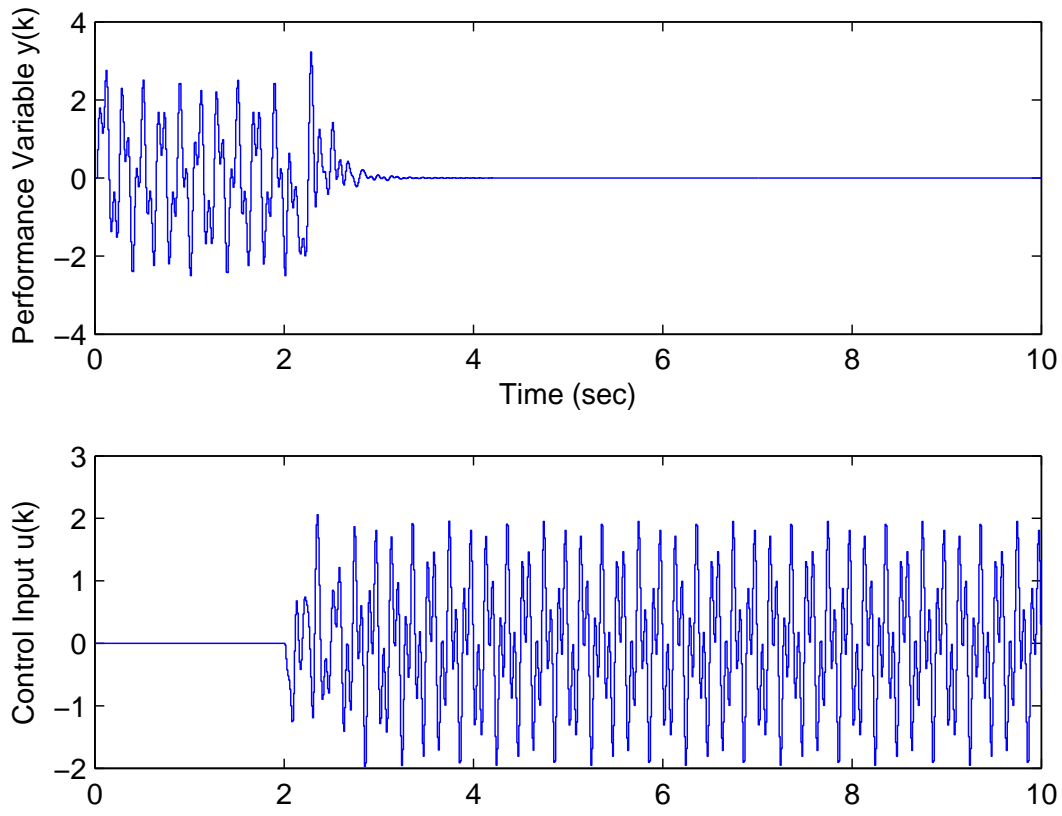

Figure 15. Closed-loop disturbance rejection response for a stable, minimum phase, relative degree $d=3$, SISO plant where the controller is created with Markov parameters perturbed by zero-mean Gaussian white noise with standard deviation $\sigma=0.25$. The control is turned on at $t=2 \mathrm{sec}$. The controller order is $n_{\mathrm{c}}=15$ with parameters $p=1, \mu=3, \alpha=25$. 
Next, consider Example IV.2 with model error in the Markov parameters. The system has relative degree $d=3$ with $H_{3}=1$. For controller implementation, we perturb each Markov parameter $H_{i}, i=1 \ldots \mu$, by adding zero-mean Gaussian white noise with standard deviation $\sigma=0.25$. We take $n_{\mathrm{c}}=15, p=1, \mu=10$, and $\alpha=25$. The closed-loop response is shown in Figure 16. The control is turned on at $t=2 \mathrm{sec}$, and the performance variable reduces to zero.
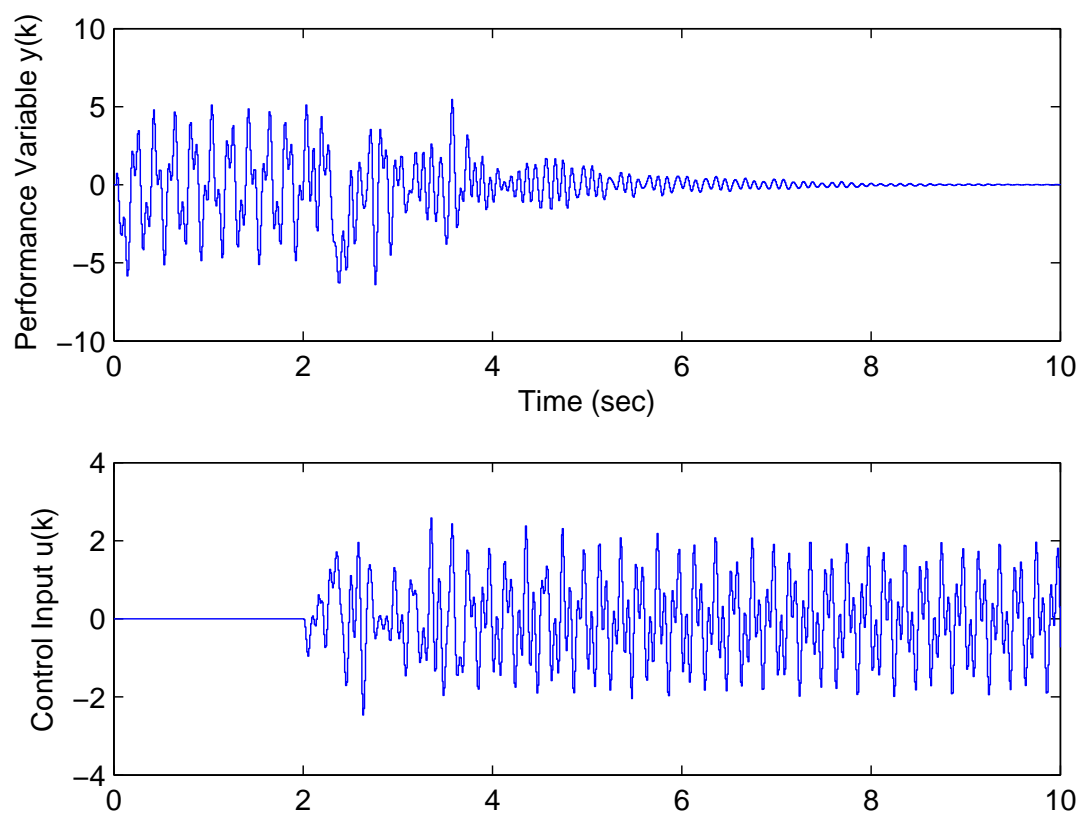

Figure 16. Closed-loop disturbance rejection response for a stable, nonminimum phase, relative degree $d=3$, SISO plant where the controller is created with Markov parameters perturbed by zero-mean Gaussian white noise with standard deviation $\sigma=0.25$. The control is turned on at $t=2 \mathrm{sec}$. The controller order is $n_{\mathrm{c}}=15$ with parameters $p=1, \mu=10, \alpha=25$.

These simulations show that the adaptive control algorithm is robust to errors in the Markov parameters.

Example V.4 (Ex. IV.1 with Noisy Measurements). To assess the performance of the adaptive algorithm with added sensor noise, we modify the sensor equation (2) by

$$
z(k)=C x(k)+D_{2} w(k)+v(k),
$$

where $v(k) \in \mathbb{R}^{l_{y}}$ is zero-mean Gaussian white noise with standard deviation $\sigma=0.1$.

We take $n_{\mathrm{c}}=15, p=1, \mu=3$, and $\alpha=25$. The closed-loop response is shown in Figure 17 . The control is turned on at $t=2 \mathrm{sec}$, and the performance variable is reduced to the level of the additive sensor noise $v(k)$. Analogous results are obtained for sinusoidal sensor noise and measurement bias, that is, constant measurement noise. Bursting was not observed in any of the simulations.

Example V.5 (Ex. IV.1 with Actuator and Sensor Saturation). In addition to the issues discussed above, physical systems are constrained by actuator and sensor limitations. In particular, we consider the performance of the adaptive algorithm under actuator and sensor saturation.

The control input $u(k)$ is subject to saturation at \pm 1.5 , while the sensor measurement $y(k)$ is subject to saturation at \pm 2 . We take $n_{\mathrm{c}}=15, p=1, \mu=3$, and $\alpha=25$. The closed-loop response is shown in Figure 18. The control is turned on at $t=2 \mathrm{sec}$, and the performance variable is reduced to a level consistent with what the saturated control can provide.

Example V.6 (Ex. IV.1 Command Following with Actuator Saturation). We consider a command given by $w(k)=1$. With the plant realized in controllable canonical form, we take $D_{1}=0$ and $E_{0}=-1$. 

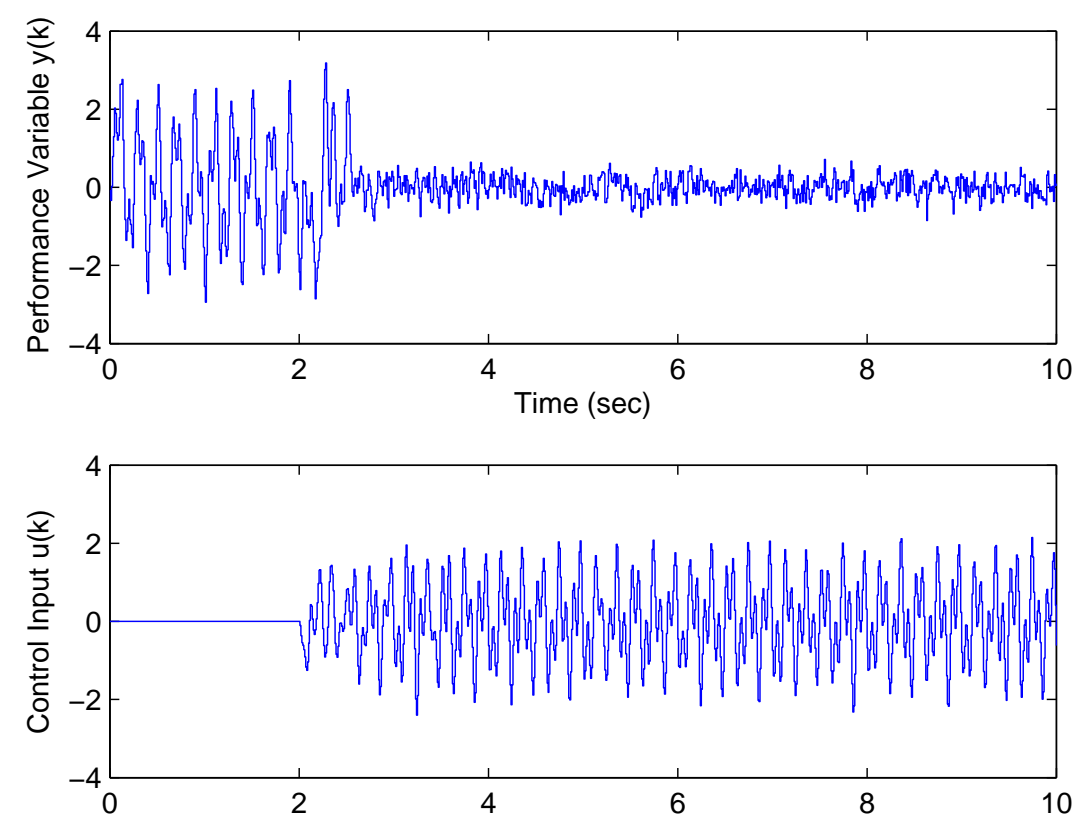

Figure 17. Closed-loop disturbance rejection response for a stable, minimum phase, SISO plant with random white noise added to the measurement. The control is turned on at $t=2 \mathrm{sec}$. The controller order is $n_{\mathrm{c}}=15$ with parameters $p=1, \mu=3, \alpha=25$. The performance variable $y(k)$ is reduced to the level of the additive sensor noise $v(k)$.
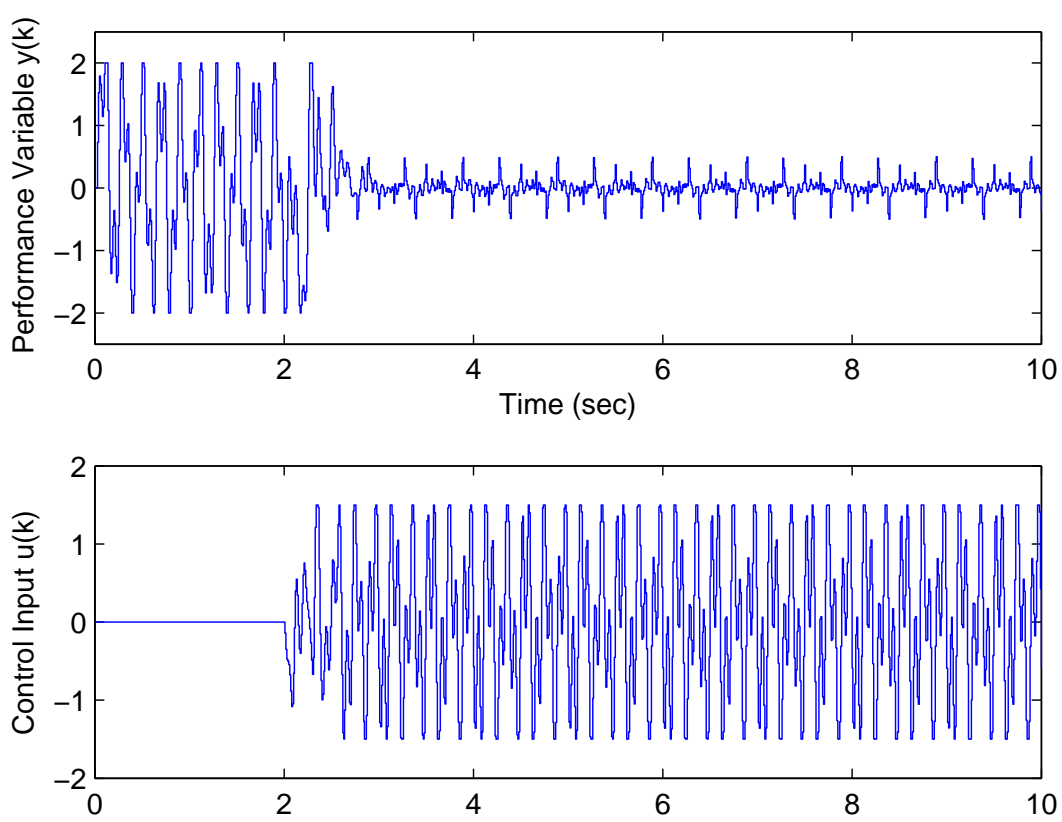

Figure 18. Closed-loop disturbance rejection response for a stable minimum phase SISO plant where the actuator is saturated at \pm 1.5 and the sensor is saturated at \pm 2 . The control is turned on at $t=2$ sec. The controller order is $n_{\mathrm{c}}=15$ with parameters $p=1, \mu=3, \alpha=25$. The saturations reduce overall steady-state performance.

First, consider the case with no actuator saturation. We take $n_{\mathrm{c}}=15, p=1, \mu=3$, and $\alpha=25$. The closed-loop response is shown in Figure 19. The control is turned on at $t=2 \mathrm{sec}$, and, after a transient, the performance variable reduces to zero, that is, the step-command $w$ is followed. 

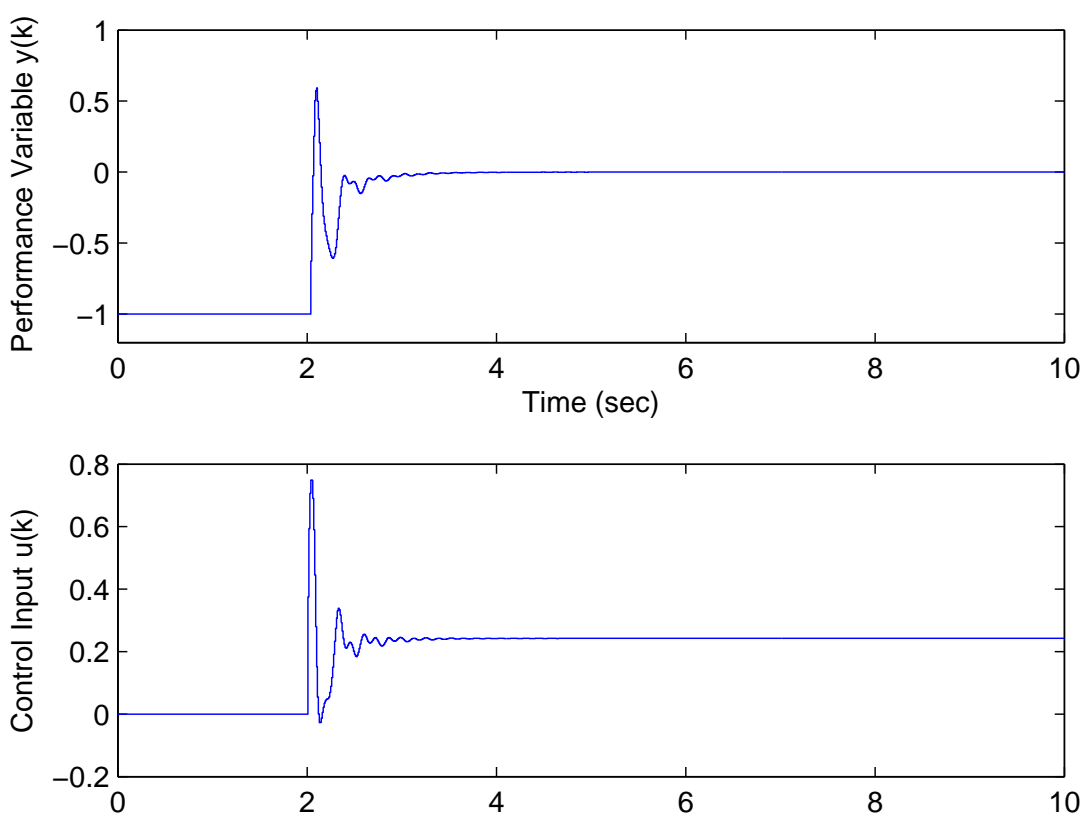

Figure 19. Closed-loop response for a stable, minimum phase, SISO plant with a step command. The control is turned on at $t=2 \mathrm{sec}$. The controller order is $n_{\mathrm{c}}=15$ with parameters $p=1, \mu=3, \alpha=25$.

Now, consider the case with actuator saturation at \pm 0.1 . We take $n_{\mathrm{c}}=15, p=1, \mu=3$, and $\alpha=25$. The closed-loop response is shown in Figure 20. The control is turned on at $t=2 \mathrm{sec}$, and the performance variable reduces to a level consistent with what the saturated control can provide.
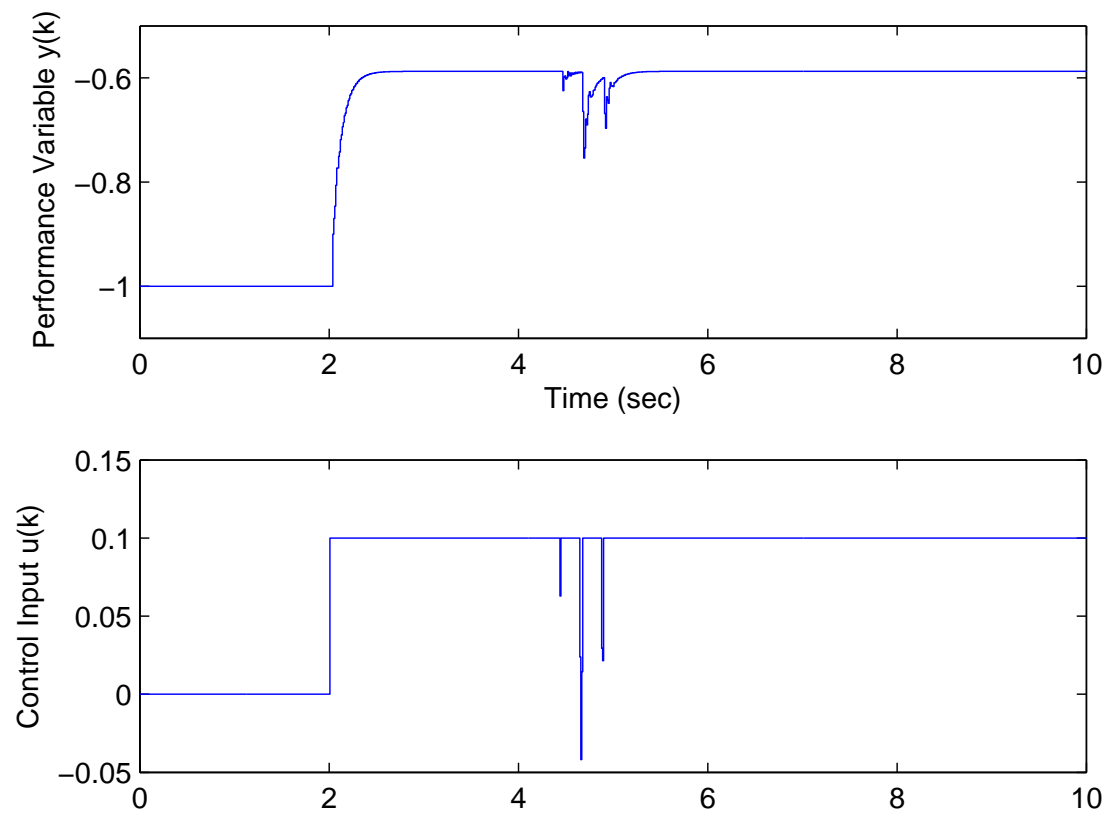

Figure 20. Closed-loop response for a stable, minimum phase, SISO plant with a step command subject to actuator saturation at \pm 0.1 . The control is turned on at $t=2 \mathrm{sec}$. The controller order is $n_{\mathrm{c}}=15$ with parameters $p=1, \mu=3, \alpha=$ 25. 


\section{Numerical Examples - Model Reference Adaptive Control}

We now present a numerical example to illustrate the response of the RCF adaptive control algorithm for model reference adaptive control (see Figure 1). Consider the longitudinal dynamics of a Boeing 747 aircraft, linearized about steady flight at 40,000 ft and $774 \mathrm{ft} / \mathrm{sec}$. The inputs to the dynamical system are taken to be elevator deflection and thrust. The output of the dynamical system is taken to be pitch angle. The continuous-time equations of motion are thus given by

$$
\begin{aligned}
{\left[\begin{array}{c}
\dot{u} \\
\dot{w} \\
\dot{q} \\
\dot{\theta}
\end{array}\right] } & =\left[\begin{array}{cccc}
-0.003 & 0.039 & 0 & -0.322 \\
-0.065 & -0.319 & 7.74 & 0 \\
0.020 & -0.101 & -0.429 & 0 \\
0 & 0 & 1 & 0
\end{array}\right]\left[\begin{array}{c}
u \\
w \\
q \\
\theta
\end{array}\right]+\left[\begin{array}{cc}
0.010 & 1 \\
-0.180 & -0.040 \\
-1.160 & 0.598 \\
0 & 0
\end{array}\right]\left[\begin{array}{l}
\delta_{\mathrm{e}} \\
\delta_{\mathrm{T}}
\end{array}\right] \\
y & =\left[\begin{array}{l}
y_{1} \\
y_{2}
\end{array}\right]=\left[\begin{array}{llll}
0 & 0 & 0 & 1 \\
0 & 0 & 0 & 0
\end{array}\right]\left[\begin{array}{l}
u \\
w \\
q \\
\theta
\end{array}\right]+\left[\begin{array}{l}
0 \\
1
\end{array}\right] w \\
z & =y_{1}-y_{\mathrm{m}},
\end{aligned}
$$

where $w$ is the exogenous command and $y_{m}$ is the output of the reference model

$$
G_{\mathrm{m}}(s)=\frac{Y_{\mathrm{m}}(s)}{W(s)}=\frac{0.0131}{s^{2}+0.16 s+0.0131}
$$

We discretize (16)-(19) using a zero-order hold and sampling time $T_{\mathrm{s}}=0.01 \mathrm{sec}$. The reference command is taken to be a $1 \mathrm{deg}$ step command in pitch angle. The controller order is $n_{\mathrm{c}}=10$ with parameters $p=1, \mu=10, \alpha=40$. The closed-loop response is shown in Figure 21. The controller is turned on immediately and the performance variable reduces to zero within about 20 sec.
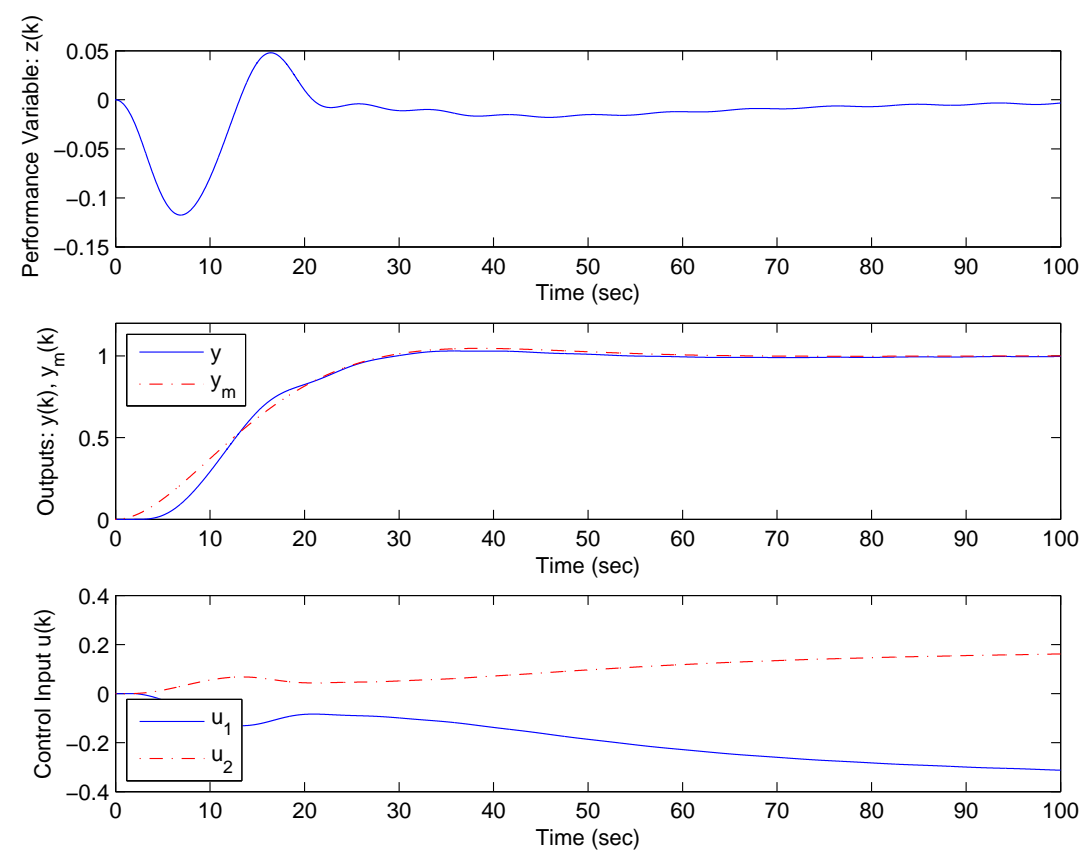

Figure 21. Closed-loop model reference adaptive control of Boeing 747 longitudinal dynamics. The controller order is $n_{\mathrm{c}}=10$ with parameters $p=1, \mu=10, \alpha=40$. The performance variable converges within about 20 sec. 


\section{Numerical Examples - Missile Longitudinal Dynamics}

We now present numerical examples for MRAC of missile longitudinal dynamics under off-nominal or damage situations. The MRAC control architecture is shown in Figure 1. The basic missile longitudinal plant $^{22}$ is derived from the short period approximation of the longitudinal equations of motion, given by

$$
\begin{aligned}
& \dot{x}=\left[\begin{array}{cc}
-1.064 & 1 \\
290.26 & 0
\end{array}\right] x+\lambda\left[\begin{array}{c}
-0.25 \\
-331.4
\end{array}\right] u, \\
& y=\left[\begin{array}{cc}
-123.34 & 0 \\
0 & 1
\end{array}\right] x+\lambda\left[\begin{array}{c}
-13.51 \\
0
\end{array}\right] u,
\end{aligned}
$$

where

$$
x \triangleq\left[\begin{array}{c}
\alpha \\
q
\end{array}\right], \quad y \triangleq\left[\begin{array}{c}
A_{z} \\
q
\end{array}\right]
$$

and $\lambda \in(0,1]$ represents the control effectiveness. Nominally $\lambda=1$.

The open-loop system (20), (21) is statically unstable. To overcome this instability, a classical three-loop autopilot ${ }^{22}$ is wrapped around the basic missile longitudinal plant. The adaptive controller then augments the closed-loop system to provide control in off-nominal cases, that is, when $\lambda<1$. The autopilot and adaptive controller inputs are denoted $u_{\text {ap }}$ and $u_{\text {ac }}$, respectively. Thus, the total control input $u=u_{\text {ap }}+u_{\text {ac }}$. The reference model $G_{\mathrm{m}}$ consists of the basic missile longitudinal plant with $\lambda=1$ and the classical three-loop autopilot. An actuator saturation of $\pm 30 \mathrm{deg}$ is included in the model, but no actuator or sensor dynamics are included.

Our goal is for the missile to follow a pitch acceleration command $w$ consisting of a 1- $g$ amplitude $1-\mathrm{Hz}$ square wave. The performance variable $z$ is the difference between the measured pitch acceleration $A_{z}$ and the reference model pitch acceleration $A_{z}^{*}$, that is, $z \triangleq A_{z}-A_{z}^{*}$. The closed-loop response is shown in Figure 22 for $\lambda=1$. Since the plant and reference model are identical in the nominal case, the adaptive control input $u_{\mathrm{ac}}=0$.
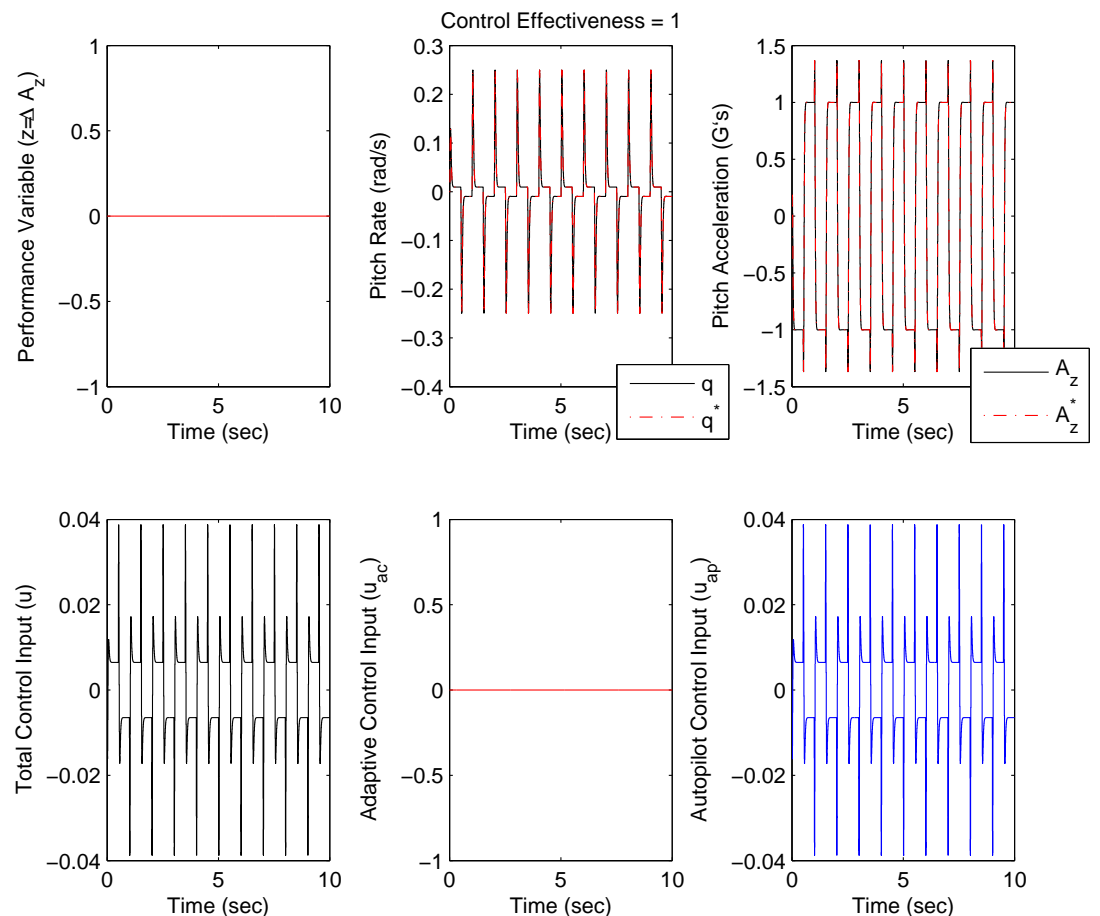

Figure 22. Closed-loop model reference adaptive control of missile longitudinal dynamics. The control effectiveness $\lambda=1$, thus the plant and reference model are identical. Therefore, the adaptive control input $u_{\mathrm{ac}}=0$ 
All of the following examples use the same adaptive controller parameters. The adaptive controller is implemented at a sampling rate of $300 \mathrm{~Hz}$. We take $n_{\mathrm{c}}=3, p=1$, and $\mu=20$. A time-varying learning rate $\alpha$ is used such that, initially, controller adaptation is fast, and, as performance improves, the adaptation slows. The learning rate is identical for each simulation. System identification using the Observer/Kalman filter identification (OKID) algorithm ${ }^{23}$ is used to obtain the 20 Markov parameters required for controller implementation. The offline identification procedure is performed with a nominal simulation $(\lambda=1)$ by injecting band-limited white noise at the adaptive controller input $u_{\text {ac }}$ and recording the performance variable $z$ while the autopilot is in-the-loop. No external disturbances are assumed to be present during the identification procedure.

Example VII.1 (75\% Control Effectiveness). Consider $\lambda=0.75$. First, Figure 23 shows simulation results with the adaptive controller turned off, that is, autopilot-only control.
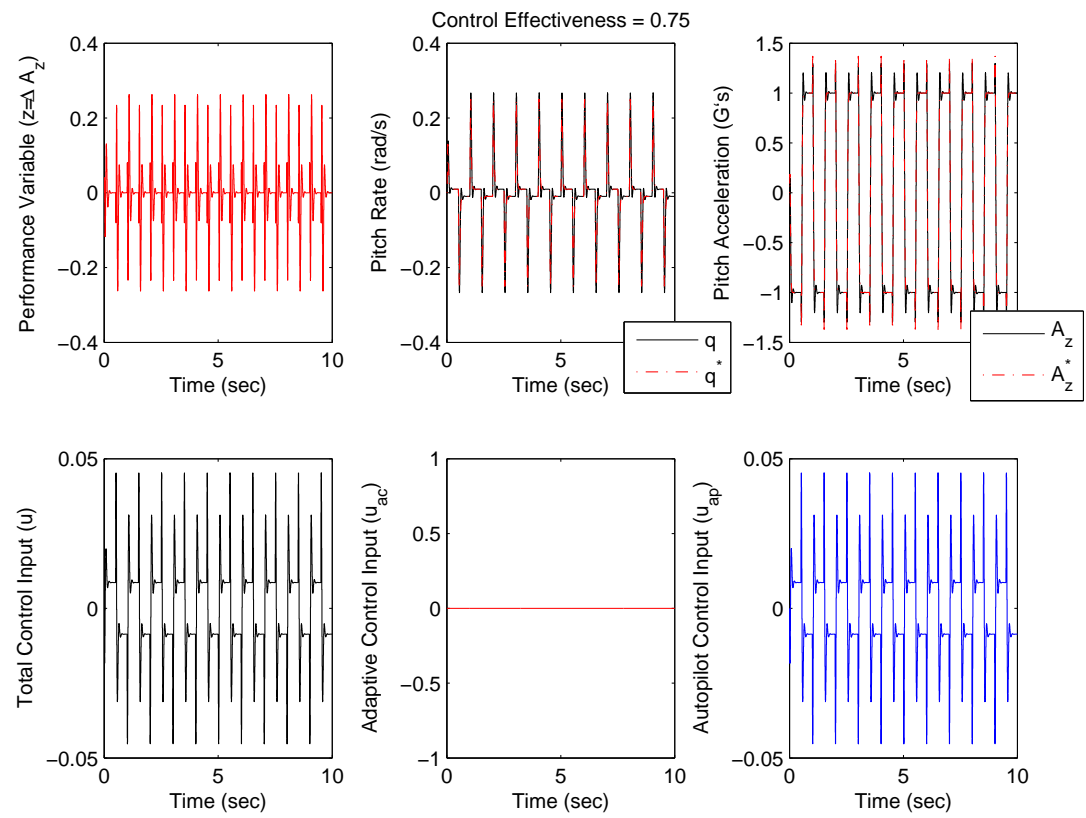

Figure 23. Missile longitudinal dynamics with control effectiveness $\lambda=0.75$ and adaptive controller turned off, that is, autopilot-only control.

Now, with the adaptive controller turned on, that is, augmented autopilot plus adaptive controller, simulation results are shown in Figure 24. After a small transient, the augmented controllers result in better performance than the autopilot-only simulation.

Example VII.2 (50\% Control Effectiveness). Consider $\lambda=0.50$. First, Figure 25 shows simulation results with the adaptive controller turned off, that is, autopilot-only control.

Now, with the adaptive controller turned on, that is, augmented autopilot plus adaptive controller, simulation results are shown in Figure 26. After a transient, the augmented controllers result in better performance than the autopilot-only simulation.

Example VII.3 (25\% Control Effectiveness). Consider $\lambda=0.25$. With the adaptive controller turned off, that is, autopilot-only control, the simulation fails. With the adaptive controller turned on, that is, augmented autopilot plus adaptive controller, simulation results are shown in Figure 27. After a transient, the augmented controllers stabilize the system whereas the autopilot-only simulation fails.

Figure 27 shows that the total control input $u$ reaches the actuator saturation level of \pm 30 deg. To reduce the initial transient, a more finely tuned learning rate can be implemented or the adaptive controller can be initialized with nonzero gains. Therefore, we now initialize the adaptive controller with the converged control gains $\theta$ from the $50 \%$ control effectiveness case. We use the gains of the $50 \%$ case since it is a median starting point. Simulation results are shown in Figure 28. The initial transient is reduced as compared with initializing the control gains to zero. In this case, the actuator saturation level is never reached. 

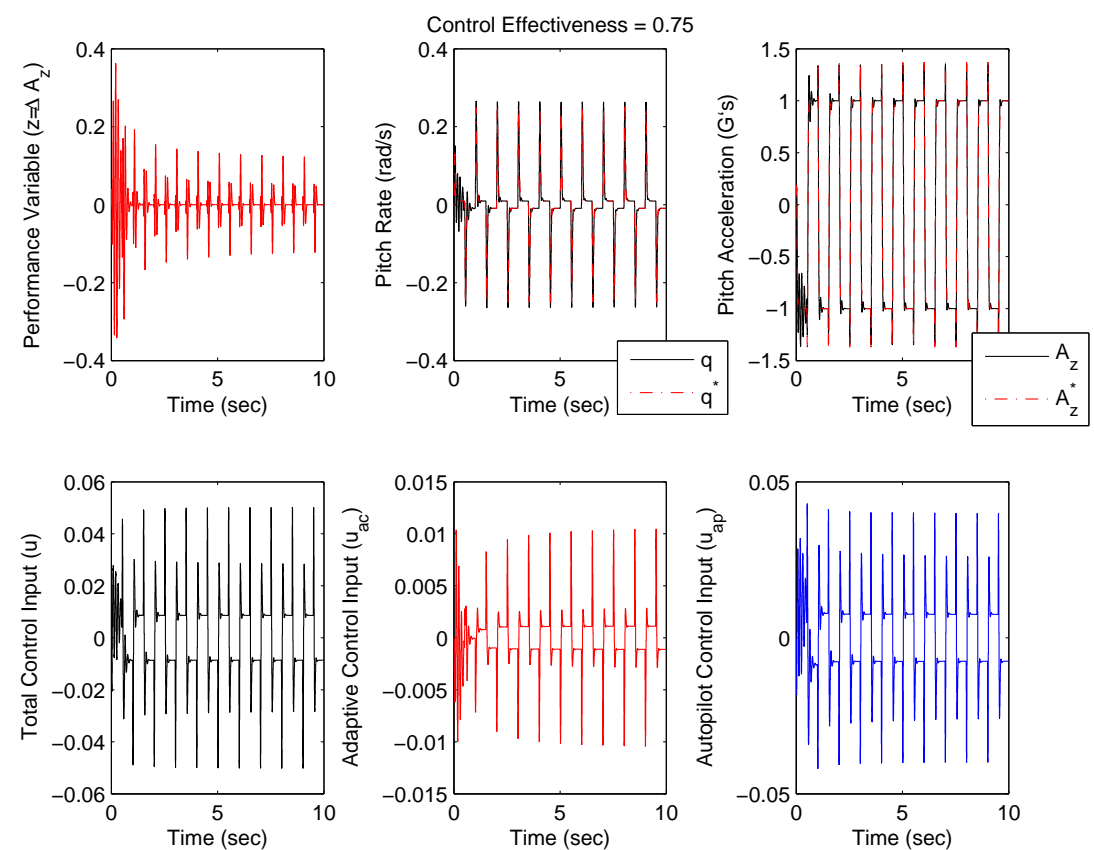

Figure 24. Closed-loop model reference adaptive control of missile longitudinal dynamics with control effectiveness $\lambda=0.75$. The augmented controllers result in better performance than the autopilot-only simulation.
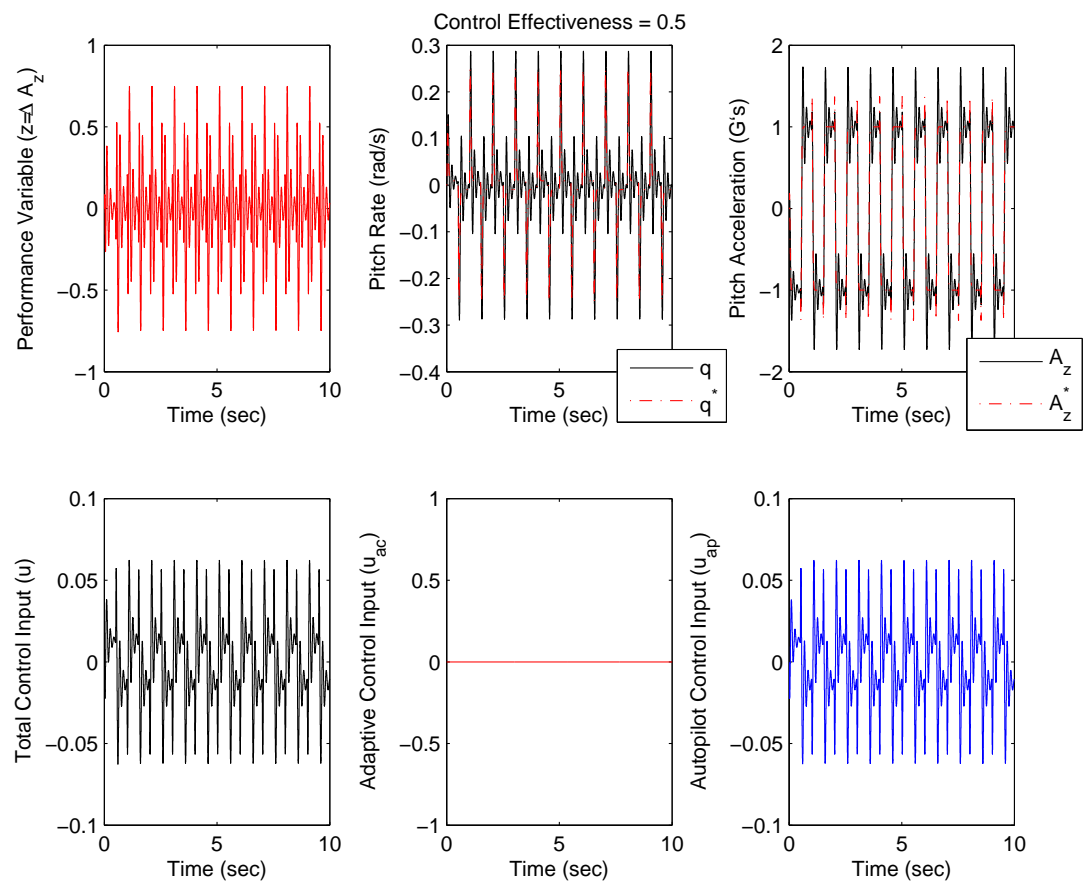

Figure 25. Missile longitudinal dynamics with control effectiveness $\lambda=0.50$ and adaptive controller turned off, that is, autopilot-only control.

\section{Conclusions}

We gave a brief overview of the RCF adaptive control algorithm and demonstrated its effectiveness in handling nonminimum-phase zeros through numerical examples illustrating the response of the algorithm

\section{9 of 22}



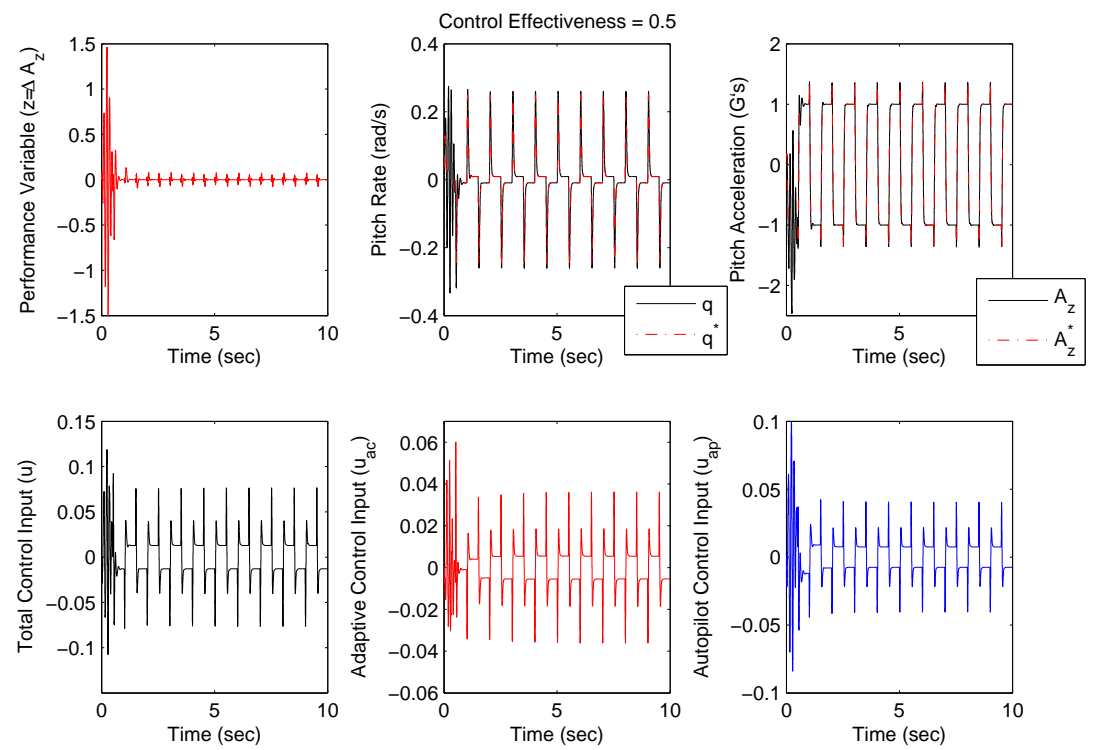

Figure 26. Closed-loop model reference adaptive control of missile longitudinal dynamics with control effectiveness $\lambda=0.50$. The augmented controllers result in better performance than the autopilot-only simulation.
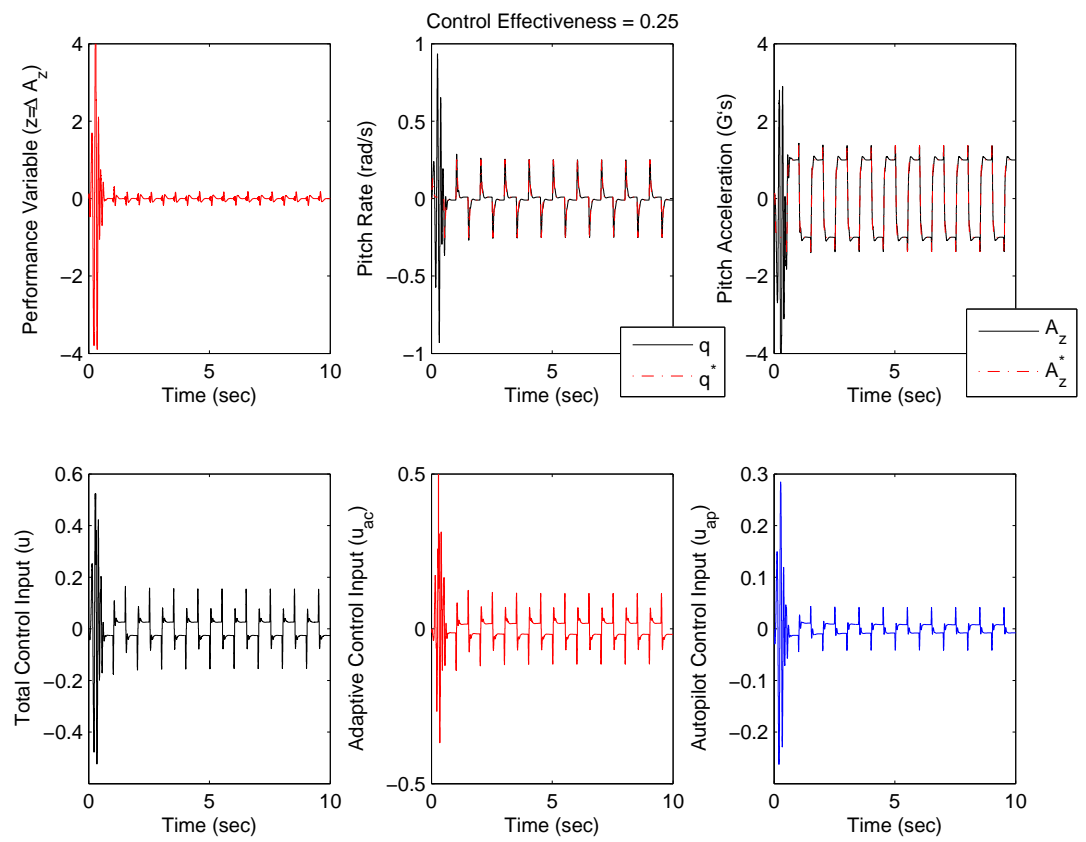

Figure 27. Closed-loop model reference adaptive control of missile longitudinal dynamics with control effectiveness $\lambda=0.25$. After a transient, the augmented controllers stabilize the system whereas the autopilot-only simulation fails. Note that the system is stabilized despite the total control input $u$ reaching the actuator saturation level of \pm 30 deg.

under modeling error in the relative degree and Markov parameters, measurement noise, and actuator and sensor saturations. We thus developed rules of thumb for choosing the learning rate $\alpha$ based on the level of model fidelity. Bursting was not observed in any of the simulations.

These numerical studies serve as guidance with regard to the development of system identification algorithms that can estimate the required plant parameters with suitable accuracy. Future work includes the development of such identification algorithms as well as Lyapunov-based stability and robustness analysis of the RCF adaptive control algorithm. 

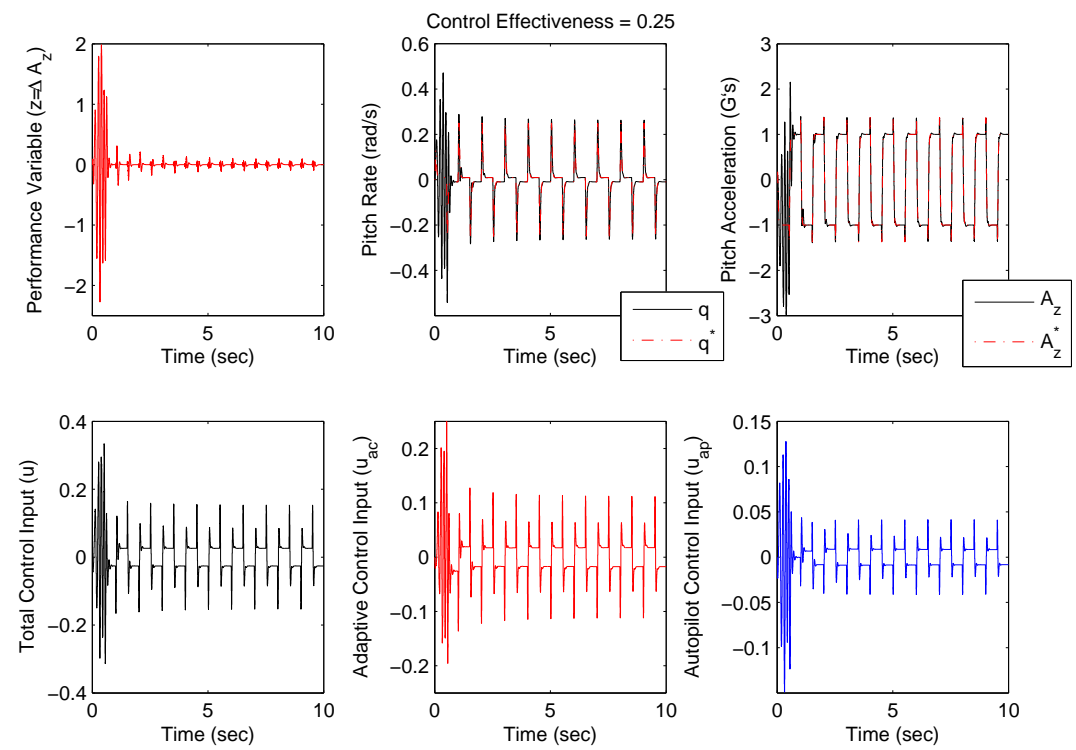

Figure 28. Closed-loop model reference adaptive control of missile longitudinal dynamics with control effectiveness $\lambda=0.25$. The adaptive controller is initialized with the converged gains from the $50 \%$ control effectiveness case. The initial transient is reduced as compared with initializing the control gains to zero. In this case, the actuator saturation level is never reached.

\section{Acknowledgments}

This research has been supported in part by the DoD under a National Defense Science and Engineering Graduate Fellowship, and NASA under IRAC Grant NNX08AB92A, Dr. S. Joshi, contract monitor.

\section{References}

${ }^{1}$ Narendra, K. S. and Annaswamy, A. M., Stable Adaptive Systems, Prentice Hall, Englewood Cliffs, New Jersey, 1989.

${ }^{2}$ Goodwin, G. C., Ramadge, P. J., and Caines, P. E., "Discrete-time multivariable adaptive control," IEEE Trans. Autom. Contr., Vol. 25, 1980, pp. 449-456.

${ }^{3}$ Hoagg, J. B., Santillo, M. A., and Bernstein, D. S., "Discrete-Time Adaptive Command Following and Disturbance Rejection with Unknown Exogenous Dynamics," IEEE Trans. Autom. Contr., Vol. 53, 2008, pp. 912-928.

${ }^{4}$ Bai, E. and Sastry, S., "Persistency of excitation, sufficient richness and parameter convergence in discrete-time adaptive control," Sys. Contr. Lett., Vol. 6, 1985, pp. 153-163.

${ }^{5}$ Rohrs, C., Valavani, L., Athans, M., and Stein, G., "Robustness of continuous-time adaptive control algorithms in the presence of unmodeled dynamics," IEEE TAC, Vol. 30, 1985, pp. 881-889.

${ }^{6}$ Ioannou, P. and Sun, J., Robust Adaptive Control, Prentice Hall, 1996.

${ }^{7}$ Ohkawa, F. and Yonezawa, Y., "A discrete model reference adaptive control system for a plant with input amplitude constraints," Int. J. Contr., Vol. 36, 1982, pp. 747-753.

${ }^{8}$ Zhang, C. and Evans, R., "Amplitude constrained adaptive control," Int. J. Contr., Vol. 46, 1987, pp. 53-64.

${ }^{9}$ Karason, S. and Annaswamy, A., "Adaptive control in the presence of input constraints," IEEE TAC, Vol. 39, 1994, pp. 2325-2330.

${ }^{10}$ Lai, W. and Cook, P., "A discrete-time universal regulator," Int. J. Contr., Vol. 62, 1995, pp. 17-32.

${ }^{11}$ Lindquist, A. and Yakubovich, V., "Universal regulators for optimal tracking in discrete-time systems affected by harmonic disturbances," IEEE Trans. Autom. Contr., Vol. 44, 1999, pp. 1688-1704.

${ }^{12}$ Anderson, B., "Topical Problems of Adaptive Control," Proc. European Contr. Conf., Kos, Greece, July 2007, pp. 4997-4998.

${ }^{13}$ Goodwin, G. C. and Sin, K. S., Adaptive Filtering, Prediction, and Control, Prentice Hall, 1984.

${ }^{14}$ Johansson, R., "Global Lyapunov stability and exponential convergence of direct adaptive control," Int. J. Contr., Vol. 50, 1989 , pp. 859-869.

${ }^{15}$ Mareels, I. and Polderman, J. W., Adaptive Systems: An Introduction, Birkhäuser, Boston, MA, 1996.

${ }^{16}$ Hayakawa, T., Haddad, W. M., and Leonessa, A., "A Lyapunov-based adaptive control framework for discrete-time non-linear systems with exogenous disturbances," Int. J. Contr., Vol. 77, 2004, pp. 250-263.

${ }^{17}$ Akhtar, S. and Bernstein, D. S., "Lyapunov-stable discrete-time model reference adaptive control," Int. J. Adaptive Contr. Signal Proc., Vol. 19, 2005, pp. 745-767. 
${ }^{18}$ Hoagg, J. B., Santillo, M. A., and Bernstein, D. S., "Internal Model Control in the Shift and Delta Domains," IEEE Trans. Autom. Contr., Vol. 53, 2008, pp. 1066-1072.

${ }^{19}$ Bayard, D. S., "Extended horizon liftings for stable inversion of nonminimum-phase systems," IEEE Trans. Autom. Contr., Vol. 39, 1994, pp. 1333-1338.

${ }^{20}$ Venugopal, R. and Bernstein, D. S., "Adaptive disturbance rejection using ARMARKOV/Toeplitz Models," IEEE Trans. Contr. Sys. Tech., Vol. 8, 2000, pp. 257-269.

${ }^{21}$ Santillo, M. A. and Bernstein, D. S., "A Retrospective Correction Filter for Discrete-Time Adaptive Control of Nonmiminum-Phase Systems," Proc. Conf. Dec. Contr., Cancun, Mexico, 2008, (to appear).

${ }^{22}$ Mracek, C. and Ridgely, D., "Missile Longitudinal Autopilots: Connections Between Optimal Control and Classical Topologies," AIAA Guidance, Navigation, and Control Conference and Exhibit, San Francisco, CA, Aug. 15-18, 2005, AIAA2005-6381.

${ }^{23}$ Juang, J. N., Applied System Identification, Prentice-Hall, Upper Saddle River, NJ, 1993. 